\title{
A Relay Strategy Actuates Pre-Existing Trisubstituted Olefins in Monoterpenoids for Cross-Metathesis with Trisubstituted Alkenes
}

\author{
Karim A. Bahou, D. Christopher Braddock,* Adam G. Meyer, G. Paul Savage, Zhensheng Shi,
} and Tianyou $\mathrm{He}$

Cite This: J. Org. Chem. 2020, 85, 4906-4917

Read Online

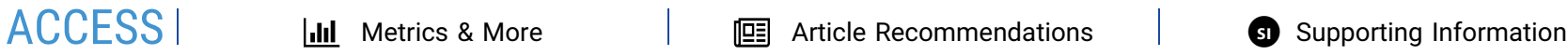

ABSTRACT: A retrosynthetic disconnection-reconnection analysis of epoxypolyenes-substrates that can undergo cyclization to podocarpanetype tricycles-reveals relay-actuated $\Delta^{6,7}$-functionalized monoterpenoid alcohols for ruthenium benzylidene catalyzed olefin cross-metathesis with homoprenyl benzenes. Successful implementation of this approach provided several epoxypolyenes as expected $(E / Z$, ca. $2-3: 1)$. The method is further generalized for the cross-metathesis of pre-existing trisubstituted olefins in other relay-actuated $\Delta^{6,7}$-functionalized monoterpenoid alcohols with various other trisubstituted alkenes to form new trisubstituted olefins. Epoxypolyene cyclization of an enantiomerically pure, but geometrically impure,

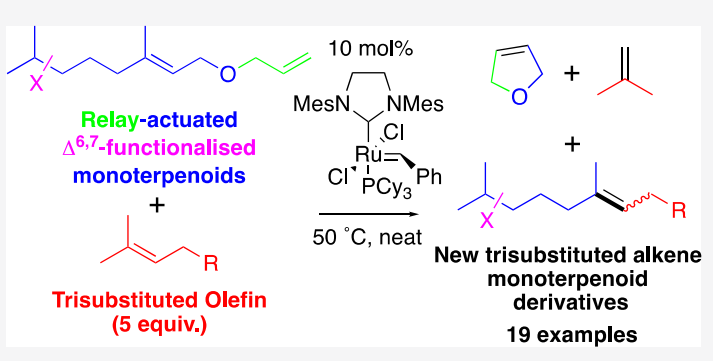
epoxypolyene substrate provides an enantiomerically pure, trans-fused, podocarpane-type tricycle (from the E-geometrical isomer).

\section{INTRODUCTION}

Biomimetically inspired polyene cyclizations have emerged as a powerful synthetic strategy for the stereocontrolled construction of complex polycarbocyclic scaffolds of biological significance, ${ }^{1}$ where epoxypolyene cyclizations of terminally functionalized geranyl units with nucleophilic aromatic headgroups have provided synthetic access to podocarpane-type tricyclic diterpene skeleta (Figure 1a). ${ }^{2}$ Such cyclization substrates are typically constructed in two steps via metalcatalyzed cross-coupling methodology of an electrophilic geranyl species in conjunction with a benzylic organometallic, and-either before or after $\mathrm{C}-\mathrm{C}$ bond constructionregioselective functionalization of the geranyl alkene at the terminus of the chain (Figure 1a). ${ }^{3}$ Each of these steps is subject to a potential disadvantage: the former is subject to competing allylic $\mathrm{S}_{\mathrm{N}} 2^{\prime}$ substitution, and the latter to nonperfect regioselective oxidation, regardless of the order of implementation. ${ }^{4}$ During the course of our studies, we had reason to consider an alternative disconnection of such functionalized linear monoterpenoid derivatives by olefin cross-metathesis, but of the two terminal olefin species that are revealed, the epoxide-containing component is synthetically nonsimplified (Figure 1b). Nonetheless, a "reconnection" operation ${ }^{5}$ reveals a geraniol derivative with a pre-existing trisubstituted olefin that we expected could be actuated for cross-metathesis by the application of Hoye's relay strategy. ${ }^{6}$ For reasons outlined below, we also elected to "reconnect" the terminal alkene component from the initial disconnection as a trisubstituted alkene.

The catalyst(s) of choice for the above proposition would be the commercially available well-defined ruthenium benzyli- denes as developed by Grubbs. ${ }^{7}$ Such catalysts are widely used to accomplish the ring-closing metathesis of disubstituted, trisubstituted, and even tetrasubstituted olefins. ${ }^{8}$ In contrast, and quite surprisingly, there are only limited reports on the formation of unfunctionalized trisubstituted olefins (as required here) by cross-metathesis using ruthenium benzylidene pre-catalysts. ${ }^{9}$ Grubbs and co-workers initially showed that ruthenium pre-catalyst $\mathbf{1}$ was competent for the crossmetathesis of geminally disubstituted olefins with terminal olefins (Figure 1c). ${ }^{10,11}$ Subsequently, Robinson and coworkers showed that the cross-metathesis of sterically challenging allyl branched 1,1-disubstituted olefins performed considerably better using a (terpenoid) prenyl rather than an allyl partner using precatalyst $\mathbf{2}$ (Figure $1 \mathrm{~d}$ ). ${ }^{12}$ With this latter literature precedent in mind, we therefore selected trisubstituted olefins as the cross-metathesis partners (Figure 1b, reconnection). ${ }^{13}$ As envisioned, this overall stratagem not only opens up the possibility of an alternative, modular, synthetic route to such cyclization precursors, but perhaps more significantly could provide a general approach to the functionalization of pre-existing trisubstituted olefins in acyclic monoterpenoid alcohols by cross-metathesis (Figure le). ${ }^{14}$ Herein, we report the success of this unprecedented olefinolefin combination to form new unfunctionalized trisubstituted

Received: January 10, 2020

Published: March 19, 2020 
SYNTHESIS OF EPOXYPOLYENE CYCLIZATION SUBSTRATES

a) Previous work:

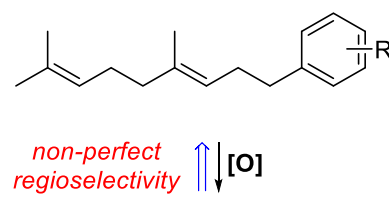

regioselectivity $[\mathbf{[}]$

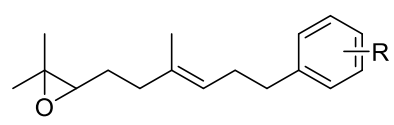

Generic substrate for epoxypolvene cyclizations

b) This work:

$\| \uparrow$ Cross-metathesis

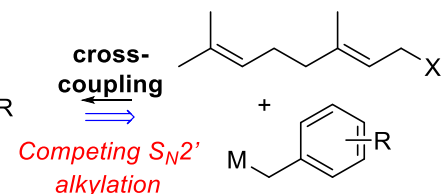
alkylation

Epoxypolyene cyclization conditions

$\overrightarrow{\text { (ref. 2) }}$

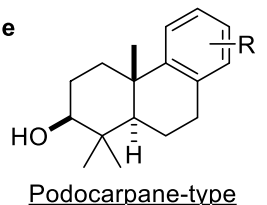
diterpenoids

SYNTHESIS OF TRISUBSTITUTED OLEFINS BY CROSS METATHESIS

c) Previous work by Grubbs:

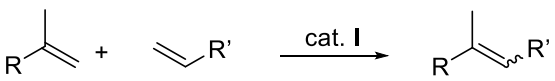

Non-terpenoid SMs

d) Previous work by Robinson:

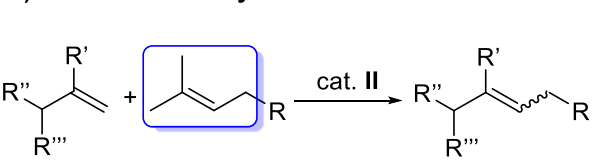

One terpenoid $\underline{\mathrm{SM}}$
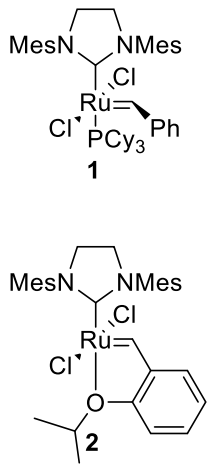

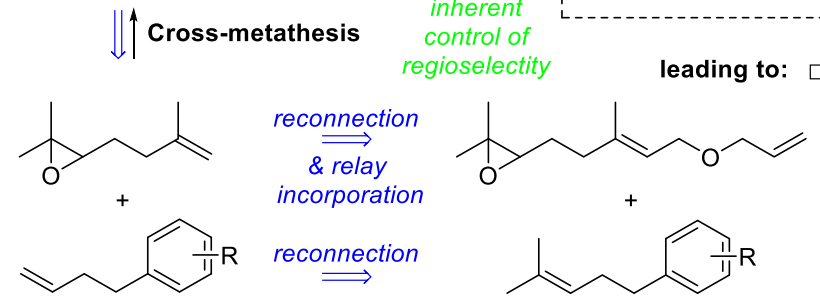

e) Actuation of Monoterpenoid Olefins for Cross Metathesis

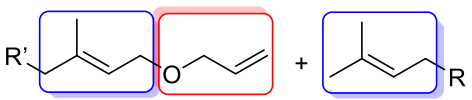

Both terpenoid SM

Figure 1. (a) Representative epoxypolyene cyclization and previous generic synthetic approach; (b) proposed alternative disconnection, reconnections, and relay incorporation; $(c, d)$ previous ruthenium benzylidene-catalyzed cross-metathesis reactions to produce unfunctionalized trisubstituted olefins; (e) this work.

olefins by cross-metathesis (Figure 1e), where the overall transformation can be classified as a relay cross-metathesis. This relay cross-metathesis reaction ("ReXM") distinguishes itself from the very limited literature precedent for such reactions by being the first such example to form isolated, unconjugated, trisubstituted alkenes where all previous reports have formed conjugated alkenes. ${ }^{15,16}$

\section{RESULTS AND DISCUSSION}

We commenced our investigations with two main objectives in mind: (i) demonstration of proof-of-principle ReXM of monoterpenoid alcohol derivatives with homoprenylbenzenes to prepare representative epoxypolyene cyclization substrates; (ii) exemplification of the method as a general approach for the functionalization of pre-existing trisubstituted olefins in acyclic monoterpenoid alcohols. Accordingly, we assembled relaymodified $\Delta^{6,7}$-functionalized monoterpenes $(E)$-5a and $(Z)-5 a$ from geraniol $[(E)-3]$ and nerol $[(Z)-3]$ via allylation ${ }^{17}$ and epoxidation with $m$ CPBA (Scheme 1 ). We also prepared diols $(S)$ - and $(R)-5 \mathbf{b}$ via Sharpless dihydroxylation ${ }^{18}$ of triene $(E)-4$ in excellent enantiomeric purity-confirmed by conversion to their respective benzoates $\mathbf{5 c}$ and chiral stationary phase highperformance liquid chromatography (HPLC) analysis (see Supporting Information) - and thence acetonides (S)- and (R)-5d (Scheme 2) by ketalization. Relay-free acetonide $(S)$ 5e was prepared from geranyl acetate as a control substrate by the use of Scafato's methods. ${ }^{19}$ Control substrate $(S)-5 f$ was prepared by the action of Grubbs catalyst $\mathbf{1}$ on $(S)-\mathbf{5 d}$, thereby inherently confirming the ability of the allyl group to function as a relay in this situation. Boronates $(S)$ - and $(R)-5 \mathrm{~g}$ were also prepared from diols $(S)$ - and $(R)-5 \mathbf{b}$ by direct condensation with phenyl boronic acid in ethyl acetate. These latter substrates, now incorporating UV-active chromophores, could be analyzed directly by HPLC for enantiomeric purity and were found to have identical enantiomeric excesses to benzoates $(S)$ - and $(R)-5 c$ (see the Supporting Information).
Scheme 1. Synthesis of Relay-Modified $\Delta^{6,7}$-Functionalized Monoterpenes (E)-5a and (Z)-5a

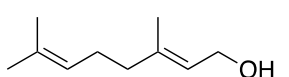

$(E)-3$

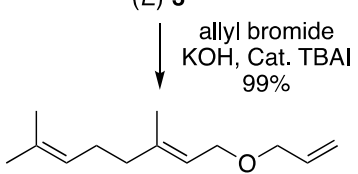

(E)-4

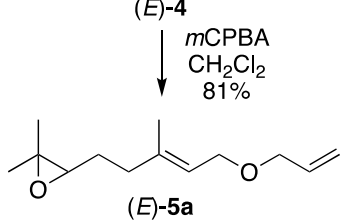

$(E)-5 \mathbf{a}$

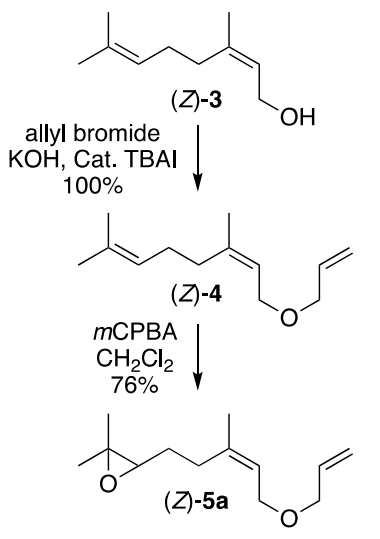

(Z)-
Attention now turned to the assembling of a collection of suitable trisubstituted alkenes for this study. Trisubstituted alkenes $7 \mathbf{a}$ and $7 \mathbf{d}-7 \mathbf{k}$ were prepared by the Wittig reaction of isopropyl phosphonium iodide (6) with aldehydes (Scheme $3)^{20}$ Alternatively, trisubstituted alkene $7 \mathrm{c}$ could be prepared by the reaction of the corresponding benzylic Grignard reagent with prenyl bromide under $\operatorname{Pd}(0)$ catalysis. $^{3 \mathrm{~b}}$ The former method is preferred, as nonperfect regioselectivity from competing $\mathrm{S}_{\mathrm{N}} 2^{\prime}$ attack is possible in the latter. Prenyl acetone $7 \mathbf{l}$ was commercially available, as was terminal alkene $7 \mathbf{b}$, which was used for control experiments (vide infra).

With these substrates in hand, we selected relay (E)-5a and trisubstituted alkene $7 \mathbf{a}$ as the partner olefin to test in the proposed ReXM reaction. It is well established that trisubstituted olefins-classified as type III olefins ${ }^{21}$-do not homodimerize, and this prompted us to use trisubstituted alkene $7 \mathbf{a}$ in excess with the expectation that this would thereby help facilitate the desired cross-metathesis. Although various attempts to mediate the proposed ReXM in toluene or 
Scheme 2. Synthesis of Various $\Delta^{6,7}$-Functionalized Monoterpenes $5 b-g$

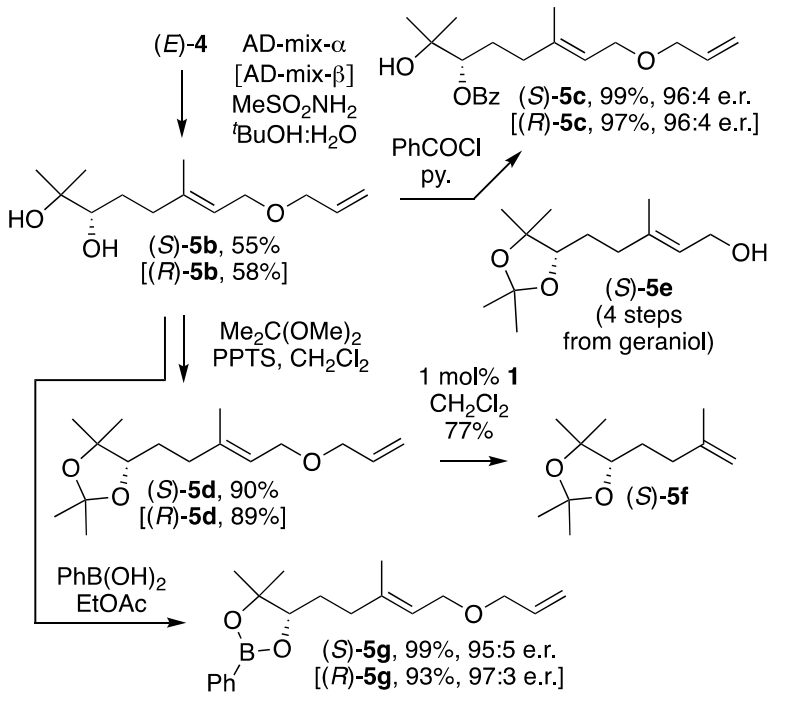

Scheme 3. Synthesis of Various Trisubstituted Alkenes

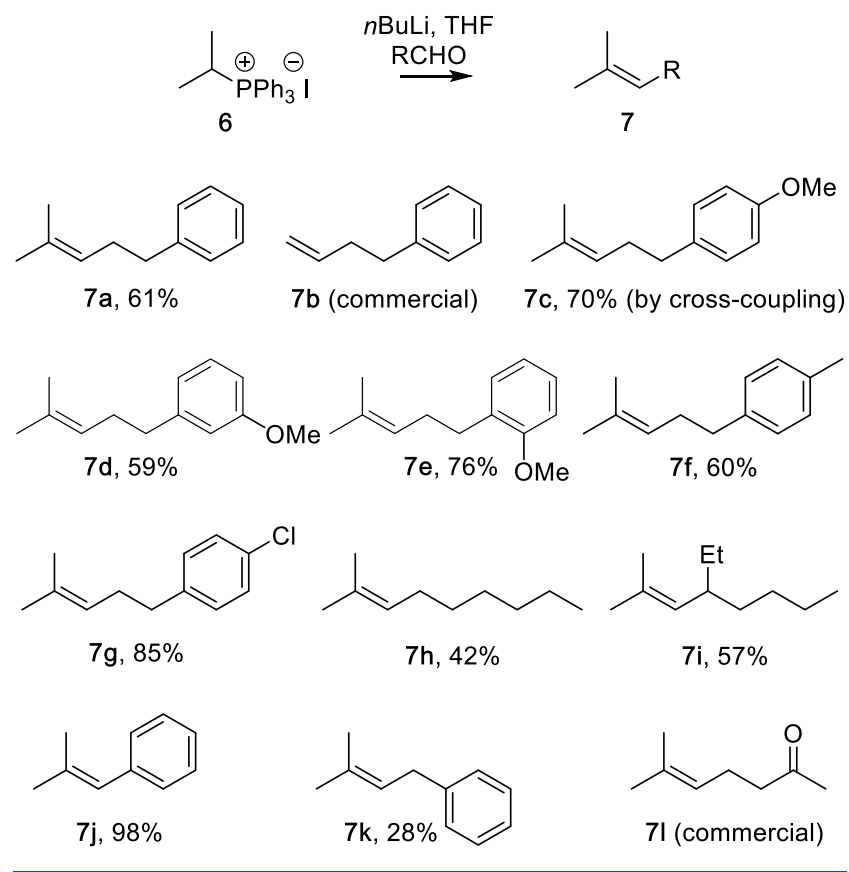

dichloromethane solution failed, neat epoxide $(E)$-5a underwent smooth ReXM using $10 \mathrm{~mol} \% 1$ with trisubstituted alkene $7 \mathrm{a}$ ( 5 equiv) at $50{ }^{\circ} \mathrm{C}$ to provide functionalized epoxypolyene 8a in excellent isolated yield (Table 1, entry 1). Surprisingly, the use of Hoveyda-Grubbs precatalyst 2 under identical conditions gave only a trace of the product $8 \mathbf{a}$ in a complex product mixture (entry 2 ), and all further metatheses were conducted with catalyst $\mathbf{1}$. In further stark contrast, the use of terminal olefin $\mathbf{7 b}$ under the same conditions (entry 3 ) with epoxide (E)-5a gave instead direct cross-metathesis product 9a and isomerized vinyl ether 10a as the major epoxide-containing products, demonstrating that the use of a trisubstituted alkene is critical for these reactions. Control experiments with acetonides (S)-5d-f (entries 5-7) ${ }^{22}$ verify also the vital role of the relay in this ReXM process, and a comparison with the reaction with $Z$-epoxide $(Z)$-5a (entry 4)
Table 1. ReXM of Relay-Actuated $\Delta^{6,7}$-Functionalized Monoterpenoids with Homoprenyl Benzenes Using $10 \mathrm{~mol}$ \% GII Catalyst (1)

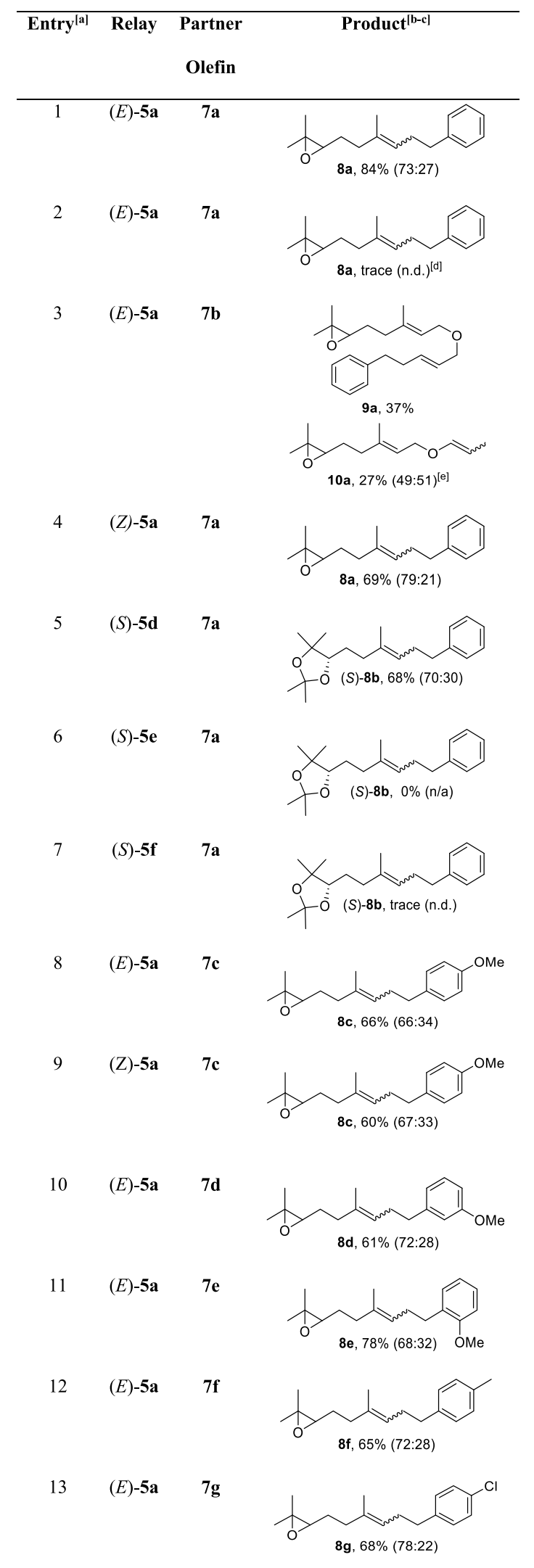

${ }^{a} 0.25 \mathrm{mmol}$ scale, conditions: olefin (5 equiv), GII (1) (10 mol \%), neat, $50{ }^{\circ} \mathrm{C}, 1 \mathrm{~h} .{ }^{b}$ Percentage isolated yields shown after 


\section{Table 1. continued}

chromatography. ${ }^{c}$ Figures in parentheses are the $E / Z$ ratio determined by ${ }^{1} \mathrm{H}$ NMR and assigned on the basis of characteristic ${ }^{13} \mathrm{C}$ NMR shielded methyl resonances for E-isomers (see the Experimental Section for details) ${ }^{d}$ Hoveyda-Grubbs II catalyst 2 (10 mol \%) employed ${ }^{e} E / Z$ ratio determined by ${ }^{1} \mathrm{H}$ NMR and assigned on the basis of characteristic ${ }^{3} \mathrm{~J}_{\mathrm{H}-\mathrm{H}}$ coupling constants.

establishes the olefin geometry in the relay substrate as unimportant. Further examples of epoxides (E)- and (Z)-5a with various homoprenyl benzenes $7 \mathrm{c}-\mathrm{g}$ establish the generality of the method (entries 8-13). ${ }^{23}$ In all successful cases, the ReXM products 8a-g were obtained with moderate E-olefin selectivity (ca. 2-3:1), as inseparable isomers, which is a limitation of the method. ${ }^{24}$ However, these selectivities are directly comparable to those previously reported for the formation of trisubstituted olefins by cross-metathesis with ruthenium benzylidene precatalysts (cf Figure 1c,d). ${ }^{10-12}$

A possible catalytic cycle for this ReXM process using representative epoxide $(E)-5 a$ with homoprenyl benzene 7a invokes Diver ${ }^{15}$ for the conversion of $\mathbf{A}$ to $\mathbf{B}$ with loss of dihydrofuran (Figure 2). The regioselective reactions of

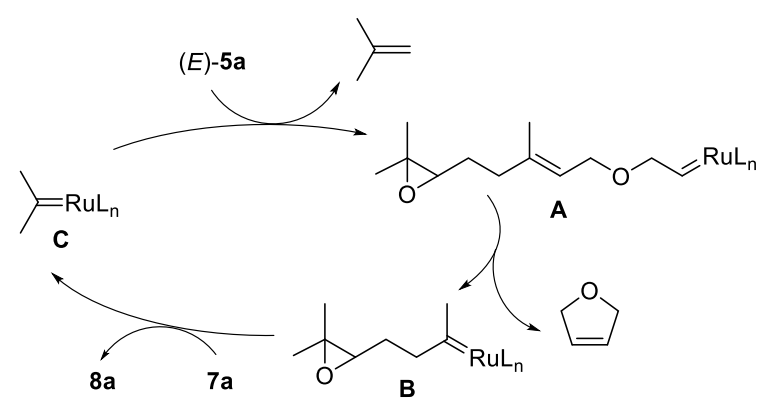

Figure 2. Possible catalytic cycle for the ReXM reaction.

ruthenium species of type $\mathbf{B}$ with trisubstituted olefins have been proposed by Robinson, ${ }^{12}$ which would produce the ReXM product 8a and ruthenium isopropylidene $\mathbf{C}$. In this scenario, the catalytic cycle would be closed by re-initiation of ruthenium isopropylidene $\mathbf{C}^{11 \mathrm{a}}$ on the terminal olefin of relay epoxide (E)-5a with concomitant loss of isobutylene. ${ }^{25}$ This mechanism is consistent also with the results obtained using nerol versus geraniol-derived substrates (cf, Table 1, entries 1 vs $4 \&$ entries 8 vs 9) as the same ruthenium alkylidene of the type $\mathbf{B}$ should be formed after initial relay metathesis.

With the ReXM method established for the reaction with homoprenyl benzenes, we explored further reactions with a variety of relay substrates and different trisubstituted alkenes as a general method for the functionalization of pre-existing trisubstituted olefins in acyclic monoterpenoid alcohols (Table 2). Thus, epoxide (E)-5a underwent smooth ReXM with aliphatic trisubstituted alkene $7 \mathbf{h}$ to give ReXM product $\mathbf{8 h}$ in excellent yield (Table 2, entry 1). $\alpha$-Branching of the alkyl chain as in olefin $7 \mathbf{i}$ (entry 2 ) proved to be detrimental to the process, where $\beta, \beta$-dimethylstyrene $(7 \mathbf{j})$ and prenylbenzene (7k) (entries 3-4) as partner olefins also failed-producing only truncated alkene $5 \mathbf{h}$-presumably on the basis of increased steric demand in each of these partner olefins. Readily available prenyl acetone $\mathbf{7 l}$ gave the ReXM product $\mathbf{8 i}$ (entry 5), but diol (S)-5b unexpectedly failed to undergo ReXM (entry 6), resulting in truncated compound $5 \mathbf{i}$ and
Table 2. ReXM of Relay-Actuated $\Delta^{6,7}$-Functionalized Monoterpenes with Various Trisubstituted Olefins Using 10 mol \% GII Catalyst

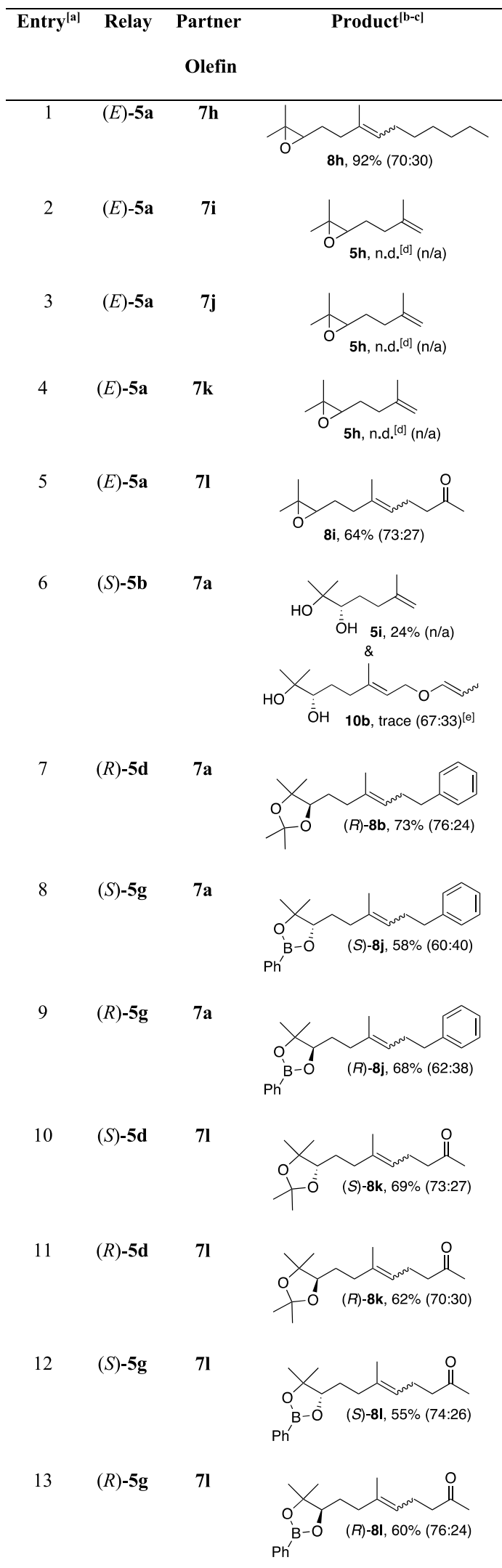

${ }^{a} 0.25 \mathrm{mmol}$ scale, conditions: olefin (5 equiv), GII (1) (10 mol \%), neat, $50{ }^{\circ} \mathrm{C}, 1 \mathrm{~h} .{ }^{b}$ Percentage isolated yields shown after 


\section{Table 2. continued}

chromatography. ${ }^{c}$ Figures in parentheses are the $E / Z$ ratio determined by ${ }^{1} \mathrm{H}$ NMR and assigned on the basis of characteristic ${ }^{13} \mathrm{C}$ NMR shielded methyl resonances for E-isomers (see the Experimental Section for details). "'Truncated" compound $5 \mathrm{~h}$ was not isolated because of its volatility but assigned on the basis of a characteristic ${ }^{1} \mathrm{H}$ resonance at $\delta 4.72(\mathrm{~m}, 2 \mathrm{H}) \mathrm{ppm} .{ }^{e} \mathrm{E} / \mathrm{Z}$ ratio determined by ${ }^{1} \mathrm{H}$ NMR and assigned on the basis of characteristic ${ }^{3} J_{\mathrm{H}-\mathrm{H}}$ coupling constants.

isomerized product $\mathbf{1 0 b}$ (implicating catalyst decomposition to a ruthenium hydride species). ${ }^{26}$ Acetonides $(S)-\&(R)-5 \mathbf{d}$ and boronates $(S)-\&(R)-\mathbf{5 g}$, however, participated cleanly in ReXM reactions (entries 7-13) to provide the desired products $(S)-\&(R)-\mathbf{8 b}, \mathbf{8} \mathbf{j}-\mathbf{l}$ without complication. In these latter instances, these substrates are all derived from highly enantioselective Sharpless dihydroxylations of geranyl allyl ether [(E)-4, vide infra], thereby providing the ReXM adducts in uniformly high enantiomeric excess, which we flag as an advantage of this methodology.

In order to overcome the inherent $E / Z$ mixture limitation of this cross-metathesis method, we elected to demonstrate an epoxypolyene cyclization with the expectation that any resulting products would have more marked polarity differences. Accordingly, we prepared ReXM product $(R)-8 \mathrm{c}$ from enantiomerically pure epoxide $(R, E)-5 \mathbf{a}$ and homoprenyl methoxybenzene $(7 c)$ in good yield $(81 \%)$ as an inseparable 2:1 E/Z mixture (Scheme 4). Boron trifluoride-promoted

\section{Scheme 4. ReXM-Epoxypolyene Cyclization Sequence}

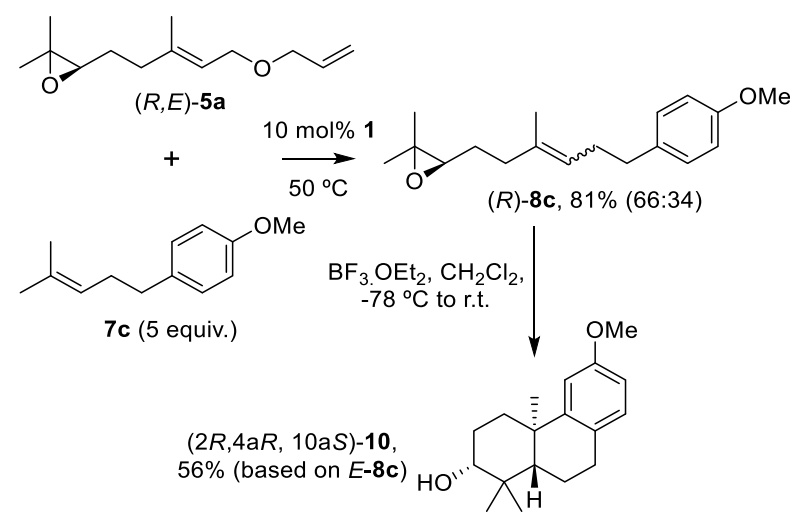

epoxypolyene cyclization of this $E / Z$ mixture provided single enantiomer podocarpane-type tricycle 11 (56\% yield based on $E-8 c)$ as a single diastereoisomer, which was readily separated away from the other components in the reaction mixture. ${ }^{27}$ To the best of our knowledge, tricycle $\mathbf{1 1}$ has not previously been prepared in a single enantiomer form, ${ }^{28}$ thereby validating the utility of this two-step metathesis-cyclization sequence. ${ }^{29}$

\section{CONCLUSIONS}

In conclusion, we have designed and demonstrated a novel ruthenium benzylidene-catalyzed relay cross-metathesis ("ReXM") reaction for the preparation of podocarpane-type epoxypolyene cyclization substrates from relay-actuated $\Delta^{6,7}$. functionalized monoterpenoid alcohols with homoprenyl benzenes. It constitutes also a general method for the crossmetathesis of pre-existing trisubstituted olefins in other relayactuated $\Delta^{6,7}$-functionalized monoterpenoid alcohols with various other trisubstituted alkenes to form new trisubstituted olefins, thereby facilitating the ability to valorize terpene biomass. The limitation inherent in the method regarding $E / Z$ selectivity requires further advances in catalyst development to provide $E$ - and $Z$-selective ruthenium benzylidene catalysts for trisubstituted olefins. However, in this situation, this can be overcome by cyclization of a $E / Z$-epoxypolyene substrate to give a separable, enantiomerically pure, podocarpane-type tricycle (from the E-geometrical isomer) in comparable yield to such cyclizations already reported in the literature. ${ }^{2}$

\section{EXPERIMENTAL SECTION}

Experimental Techniques. All reactions were carried out in oven-dried glassware. Air-sensitive reactions were performed under a positive pressure of nitrogen unless stated otherwise. Reaction temperatures other than room temperature were achieved using an oil bath, ice/water bath, or dry ice/acetone. "Concentrated" refers to concentrating of the solution in vacuo. "Chromatographed" refers to flash column chromatography on silica gel, particle size 33-70 or 40$63 \mu \mathrm{m}$, unless otherwise stated. "DCVC" refers to dry column vacuum chromatography on silica gel, particle size $33-70 \mu \mathrm{m} .{ }^{30}$ Analytical thin-layer chromatography was performed on silica gel 60 F254 precoated aluminum-backed plates and visualized with either irradiation with UV light $(254 \mathrm{~nm})$ or potassium permanganate, vanillin, or phosphomolybdic acid staining. Brine refers to a saturated aqueous $\mathrm{NaCl}$ solution.

Characterization. Fourier transform infrared (IR) spectra were recorded neat using an attenuated total reflection (ATR)-IR spectrometer and absorptions are reported to the nearest wavenumber. The (expected) very weak $\mathrm{C}=\mathrm{C}$ and $\mathrm{sp}^{2} \mathrm{C}-\mathrm{H}$ bond stretches for trisubstituted alkenes $\mathbf{7}$ and $\mathbf{8}$ failed to be automatically pick peaked because they fell under the peak picking threshold, although they can be observed (in most cases) by careful inspection of the spectra. ${ }^{29}{ }^{1} \mathrm{H}$ and ${ }^{13} \mathrm{C}$ NMR spectra were recorded on either a Bruker DRX-400 or Bruker AV-400. Chemical shifts $(\delta)$ are expressed in parts per million (ppm) relative to the residual solvent peak. ${ }^{1} \mathrm{H}$ NMR spectra were recorded at $400 \mathrm{MHz} .{ }^{13} \mathrm{C}$ NMR spectra were recorded at $101 \mathrm{MHz}$. NMR acquisitions were performed at $298 \mathrm{~K}$ unless stated otherwise. Abbreviations are: s, singlet; $d$, doublet; $t$, triplet; q, quartet; qu., quintet; $\mathrm{m}$, multiplet. High-resolution mass spectrometry (HRMS) was conducted by the Imperial College Department of Chemistry Mass Spectrometry Service.

Reagents. Allyl bromide was distilled freshly before use; otherwise all reagents were obtained from commercial suppliers and used as received.

Solvents. All reactions were carried out in anhydrous solvents. HPLC grade $\mathrm{CH}_{2} \mathrm{Cl}_{2}, \mathrm{THF}$, and EtOAc were dried by passing through a column of alumina beads. Extraction solvents, chromatography eluents ( $n$-hexane, petrol, pentanes, $\mathrm{CH}_{2} \mathrm{Cl}_{2}, \mathrm{Et}_{2} \mathrm{O}$, and EtOAc), and ${ }^{t} \mathrm{BuOH}$ were used as received. "Petrol" refers to petroleum ether $\left(40-60{ }^{\circ} \mathrm{C}\right)$. Petroleum ether $\left(40-60{ }^{\circ} \mathrm{C}\right)$, EtOAc, $\mathrm{CH}_{2} \mathrm{Cl}_{2}$, and $\mathrm{Et}_{2} \mathrm{O}$ were of GPR grade and pentanes were of HPLC grade.

(E)-1-(Allyloxy)-3,7-dimethylocta-2,6-diene [(E)-4]. ${ }^{17}$ Using a modified procedure of Rao and Senthilkumar, to a mixture of neat geraniol $[(E)-3](5.30 \mathrm{~mL}, 30 \mathrm{mmol}, 1.0$ equiv), allyl bromide $(7.8$ $\mathrm{mL}, 90 \mathrm{mmol}, 3.0$ equiv), and TBAI $(554 \mathrm{mg}, 1.50 \mathrm{mmol}, 5 \mathrm{~mol} \%)$ was added crushed $\mathrm{KOH}$ pellets $(3.37 \mathrm{~g}, 60.0 \mathrm{mmol}, 2.0$ equiv) at room temperature and the mixture was stirred for $18 \mathrm{~h}$. The crude reaction mixture was purified by loading directly onto a pad of silica gel and eluting with $n$-hexane, to give allyl ether $(E)-4(5.78 \mathrm{~g}, 29.7$ mmol, 99\%) as a colorless oil. 10.14469/hpc/5738. $R_{f} 0.60(n-$ hexane); IR (ATR, neat) 1670, $1647 \mathrm{~cm}^{-1} ;{ }^{1} \mathrm{H}$ NMR (400 MHz, $\left.\mathrm{CDCl}_{3}\right): \delta 5.93(\mathrm{ddt}, J=17.2,10.3,5.7 \mathrm{~Hz}, 1 \mathrm{H}), 5.39-5.33(\mathrm{~m}, 1 \mathrm{H})$, $5.27(\mathrm{dq}, J=17.3,1.7 \mathrm{~Hz}, 1 \mathrm{H}), 5.18(\mathrm{dq}, J=10.4,1.4 \mathrm{~Hz}, 1 \mathrm{H}), 5.13-$ $5.06(\mathrm{~m}, 1 \mathrm{H}), 4.00(\mathrm{~d}, J=6.8 \mathrm{~Hz}, 2 \mathrm{H}), 3.97(\mathrm{dt}, J=5.7,1.4 \mathrm{~Hz}, 2 \mathrm{H})$, $2.16-1.96(\mathrm{~m}, 4 \mathrm{H}), 1.68(\mathrm{~d}, J=1.3 \mathrm{~Hz}, 3 \mathrm{H}), 1.66(\mathrm{~s}, 3 \mathrm{H}), 1.60(\mathrm{~s}$, $3 \mathrm{H}) ;{ }^{13} \mathrm{C}\left\{{ }^{1} \mathrm{H}\right\}$ NMR $\left(101 \mathrm{MHz}, \mathrm{CDCl}_{3}\right): \delta 140.2,135.1,131.7$, 
124.0, 120.8, 117.0, 70.9, 66.6, 39.6, 26.4, 25.7, 17.7, 16.5; HRMS $\left(\mathrm{EI}^{+}\right) \mathrm{m} / z:[\mathrm{M}]^{\bullet+}$ calcd for $\mathrm{C}_{13} \mathrm{H}_{22} \mathrm{O}, 194.1671$; found, 194.1682.

(E)-3-(5-(Allyloxy)-3-methylpent-3-en-1-yl)-2,2-dimethyloxirane $[(E)-5 a) .{ }^{31}$ To a stirred solution of ether $(E)-4(1.41 \mathrm{~g}, 7.28 \mathrm{mmol}, 1.0$ equiv) in $\mathrm{CH}_{2} \mathrm{Cl}_{2}(30 \mathrm{~mL})$ was added dropwise a solution of $m \mathrm{CPBA}$ (1.63 g, 77\%, $7.28 \mathrm{mmol}, 1.0$ equiv) in $\mathrm{CH}_{2} \mathrm{Cl}_{2}(30 \mathrm{~mL})$ over ca. $0.5 \mathrm{~h}$ at $0{ }^{\circ} \mathrm{C}$. The mixture was allowed to warm to room temperature gradually. After a total reaction time of $18 \mathrm{~h}$, the reaction mixture was concentrated, dissolved in EtOAc $(100 \mathrm{~mL})$, and washed with a saturated aqueous $\mathrm{NaHCO}_{3}$ solution $(3 \times 50 \mathrm{~mL})$, brine $(100 \mathrm{~mL})$, dried over $\mathrm{Na}_{2} \mathrm{SO}_{4}$, concentrated, and chromatographed (20-50\% $\mathrm{Et}_{2} \mathrm{O}$ in pentanes), to give epoxide $(E)-5 \mathrm{a}(1.71 \mathrm{~g}, 8.1 \mathrm{mmol}, 81 \%)$ as a colorless oil. $10.14469 / \mathrm{hpc} / 5820 . \quad R_{f} 0.57 \quad(30 \%$ EtOAc in pentanes); IR (ATR, neat) 3075, $1670 \mathrm{~cm}^{-1}$; ${ }^{1} \mathrm{H}$ NMR (400 MHz, $\left.\mathrm{CDCl}_{3}\right): \delta 5.92(\mathrm{ddt}, J=17.5,10.5,5.7 \mathrm{~Hz}, 1 \mathrm{H}), 5.43-5.36(\mathrm{~m}, 1 \mathrm{H})$, $5.26(\mathrm{dq}, J=17.2,1.7 \mathrm{~Hz}, 1 \mathrm{H}), 5.17(\mathrm{dq}, J=10.5,1.5 \mathrm{~Hz}, 1 \mathrm{H}), 3.99$ $(\mathrm{d}, J=6.8 \mathrm{~Hz}, 2 \mathrm{H}), 3.97-3.94(\mathrm{~m}, 2 \mathrm{H}), 2.70(\mathrm{t}, J=6.2 \mathrm{~Hz}, 1 \mathrm{H})$, 2.26-2.07 (m, 2H), $1.68(\mathrm{~s}, 3 \mathrm{H}), 1.67-1.62(\mathrm{~m}, 2 \mathrm{H}), 1.29(\mathrm{~s}, 3 \mathrm{H})$, $1.25(\mathrm{~s}, 3 \mathrm{H}) ;{ }^{13} \mathrm{C}\left\{{ }^{1} \mathrm{H}\right\}$ NMR $\left(101 \mathrm{MHz}, \mathrm{CDCl}_{3}\right): \delta$ 139.2, 135.0, 121.4, 117.0, 71.1, 66.5, 64.0, 58.4, 36.2, 27.2, 24.9, 18.7, 16.5; HRMS $\left(\mathrm{CI}^{+}\right) \mathrm{m} / z:[\mathrm{M}-\mathrm{H}]^{+}$calcd for $\mathrm{C}_{13} \mathrm{H}_{21} \mathrm{O}_{2}, 209.1536$; found, 209.1535 .

(Z)-1-(Allyloxy)-3,7-dimethylocta-2,6-diene [(Z)-4]. ${ }^{17}$ Using a modified procedure of Rao and Senthilkumar, to a neat mixture of nerol $[(Z)-3]$ ( $5.30 \mathrm{~mL}, 30 \mathrm{mmol}, 1.0$ equiv), allyl bromide $(7.8 \mathrm{~mL}$, $90 \mathrm{mmol}, 3.0$ equiv), and TBAI (554 mg, $1.50 \mathrm{mmol}, 5 \mathrm{~mol} \%$ ) was added crushed $\mathrm{KOH}$ pellets $(3.37 \mathrm{~g}, 60.0 \mathrm{mmol}, 2.0$ equiv) at room temperature and the mixture was stirred for $18 \mathrm{~h}$. The crude reaction mixture was purified by loading directly onto a pad of silica gel and eluting with $n$-hexane, to give allyl ether $(Z)-4(5.81 \mathrm{~g}, 29.9 \mathrm{mmol}$, quant.) as a colorless oil. 10.14469/hpc/5741. $R_{f} 0.65$ ( $n$-hexane); IR (ATR, neat) $3091,1670 \mathrm{~cm}^{-1}$; ${ }^{1} \mathrm{H}$ NMR $\left(400 \mathrm{MHz}, \mathrm{CDCl}_{3}\right): \delta 5.92$ (ddt, $J=17.3,10.3,5.6 \mathrm{~Hz}, 1 \mathrm{H}), 5.41-5.32(\mathrm{~m}, 1 \mathrm{H}), 5.30-5.24(\mathrm{~m}$, $1 \mathrm{H})$, 5.21-5.13 (m, 1H), 5.14-5.04 (m, 1H), 4.00-3.92 (m, 4H), $2.12-2.02(\mathrm{~m}, 4 \mathrm{H}), 1.75(\mathrm{~d}, J=1.2 \mathrm{~Hz}, 3 \mathrm{H}), 1.68(\mathrm{~s}, 3 \mathrm{H}), 1.60(\mathrm{~s}$, $3 \mathrm{H}) ;{ }^{13} \mathrm{C}\left\{{ }^{1} \mathrm{H}\right\}$ NMR (101 MHz, $\left.\mathrm{CDCl}_{3}\right): \delta 140.4,135.1,131.9$, 123.9, 121.8, 116.8, 71.0, 66.3, 32.3, 26.7, 25.7, 23.5, 17.6; HRMS $\left(\mathrm{EI}^{+}\right) \mathrm{m} / z:[\mathrm{M}]^{\bullet+}$ calcd for $\mathrm{C}_{13} \mathrm{H}_{22} \mathrm{O}$, 194.1671; found, 194.1670.

(Z)-3-(5-(Allyloxy)-3-methylpent-3-en-1-yl)-2,2-dimethyloxirane $[(Z)-5 a]$. To a stirred solution of ether $(Z)-4(1.41 \mathrm{~g}, 7.28 \mathrm{mmol}, 1.0$ equiv) in $\mathrm{CH}_{2} \mathrm{Cl}_{2}(30 \mathrm{~mL})$ was added dropwise a solution of $m \mathrm{CPBA}$ (1.63 g, 77\%, $7.28 \mathrm{mmol}, 1.0$ equiv) in $\mathrm{CH}_{2} \mathrm{Cl}_{2}(30 \mathrm{~mL})$ over ca. $0.5 \mathrm{~h}$ at $0{ }^{\circ} \mathrm{C}$. The mixture was allowed to warm to room temperature gradually. After a total reaction time of $18 \mathrm{~h}$, the reaction mixture was concentrated, dissolved in EtOAc $(100 \mathrm{~mL})$, and washed with a saturated aqueous $\mathrm{NaHCO}_{3}$ solution $(3 \times 50 \mathrm{~mL})$, brine $(100 \mathrm{~mL})$, dried over $\mathrm{Na}_{2} \mathrm{SO}_{4}$, concentrated, and chromatographed (20-50\% $\mathrm{Et}_{2} \mathrm{O}$ in pentanes), to give epoxide $(Z)-5 \mathrm{a}(1.60 \mathrm{~g}, 7.6 \mathrm{mmol}, 76 \%)$ as a colorless oil. 10.14469/hpc/5742. $R_{f} 0.25$ (10\% EtOAc in $n$ hexane); IR (ATR, neat) $3075 \mathrm{~cm}^{-1}$; ${ }^{1} \mathrm{H}$ NMR (400 MHz, CDCl 3 ): $\delta$ $5.92(\mathrm{ddt}, J=17.3,10.4,5.7 \mathrm{~Hz}, 1 \mathrm{H}), 5.46-5.38(\mathrm{~m}, 1 \mathrm{H}), 5.27(\mathrm{dq}, J$ $=17.2,1.6 \mathrm{~Hz}, 1 \mathrm{H}), 5.17(\mathrm{dq}, J=10.4,1.4 \mathrm{~Hz}, 1 \mathrm{H}), 4.03-3.93(\mathrm{~m}$, $4 \mathrm{H}), 2.70(\mathrm{t}, J=6.3 \mathrm{~Hz}, 1 \mathrm{H}), 2.29-2.15(\mathrm{~m}, 2 \mathrm{H}), 1.76(\mathrm{~d}, J=1.2 \mathrm{~Hz}$, $3 \mathrm{H}), 1.71-1.54(\mathrm{~m}, 2 \mathrm{H}), 1.30(\mathrm{~s}, 3 \mathrm{H}), 1.26(\mathrm{~s}, 3 \mathrm{H}) ;{ }^{13} \mathrm{C}\left\{{ }^{1} \mathrm{H}\right\} \mathrm{NMR}$ $\left(101 \mathrm{MHz} \mathrm{CDCl}_{3}\right): \delta 139.5,134.9,122.4,117.0,71.2,66.2,63.9$, 58.4, 28.9, 27.5, 24.9, 23.4, 18.7; HRMS $\left(\mathrm{ES}^{+}\right) \mathrm{m} / z:[\mathrm{M}-\mathrm{H}]^{+}$calcd for $\mathrm{C}_{13} \mathrm{H}_{21} \mathrm{O}_{2}, 209.1542$; found, 209.1547 .

(S,E)-8-(Allyloxy)-2,6-dimethyloct-6-ene-2,3-diol [(S)-5b]. ${ }^{31}$ Using a modified procedure of Sharpless, ${ }^{18}$ to a vigorously stirred solution of $\mathrm{AD}$-mix- $\alpha\left(7.0 \mathrm{~g}, 1.4 \mathrm{~g} \mathrm{~mol}^{-1}\right)$ in ${ }^{t} \mathrm{BuOH}(75 \mathrm{~mL})$ and $\mathrm{H}_{2} \mathrm{O}(75 \mathrm{~mL})$ was added $\mathrm{MeSO}_{2} \mathrm{NH}_{2}$ (476 mg, $5.0 \mathrm{mmol}, 1.0$ equiv) followed by ether (E)-4 (972 mg, $5.0 \mathrm{mmol}, 1.0$ equiv). The reaction mixture was allowed to stir for $16 \mathrm{~h}$. The mixture was quenched by the addition of a $20 \% \mathrm{Na}_{2} \mathrm{SO}_{3}$ aqueous solution $(150 \mathrm{~mL})$ and extracted with EtOAc $(3 \times 50 \mathrm{~mL})$. The combined organics were washed with brine, dried over $\mathrm{MgSO}_{4}$, concentrated, and chromatographed (40\% EtOAc in $n$ hexane), to give diol $(S)-5 \mathbf{b}(632 \mathrm{mg}, 2.8 \mathrm{mmol}, 55 \%)$ as a colorless oil. $10.14469 / \mathrm{hpc} / 5743 . R_{f} 0.36$ (40\% EtOAc in $n$-hexane); $[\alpha]_{\mathrm{D}}^{20}$ -26.1 (c 1.0, $\mathrm{CHCl}_{3}$ ); IR (ATR, neat) 3600-3100, 3075, $1666 \mathrm{~cm}^{-1}$; ${ }^{1} \mathrm{H}$ NMR $\left(400 \mathrm{MHz}, \mathrm{CDCl}_{3}\right): \delta 5.93(\mathrm{ddt}, J=17.2,10.3,5.7 \mathrm{~Hz}$,
$1 \mathrm{H}), 5.47-5.37(\mathrm{~m}, 1 \mathrm{H}), 5.28(\mathrm{dq}, J=17.2,1.6 \mathrm{~Hz}, 1 \mathrm{H}), 5.19(\mathrm{dq}, J$ $=10.3,1.4 \mathrm{~Hz}, 1 \mathrm{H}), 4.03-3.93(\mathrm{~m}, 4 \mathrm{H}), 3.36(\mathrm{dd}, J=10.5,2.0 \mathrm{~Hz}$, $1 \mathrm{H}), 2.31(\mathrm{ddd}, J=14.7,9.7,5.2 \mathrm{~Hz}, 1 \mathrm{H}), 2.16-2.06(\mathrm{~m}, 1 \mathrm{H}), 1.99-$ $1.81($ br s, $2 \mathrm{H}), 1.68(\mathrm{~s}, 3 \mathrm{H}), 1.66-1.56(\mathrm{~m}, 1 \mathrm{H}), 1.51-1.39(\mathrm{~m}$, $1 \mathrm{H}), 1.20(\mathrm{~s}, 3 \mathrm{H}), 1.16(\mathrm{~s}, 3 \mathrm{H}) ;{ }^{13} \mathrm{C}\left\{{ }^{1} \mathrm{H}\right\}$ NMR $\left(101 \mathrm{MHz}, \mathrm{CDCl}_{3}\right)$ : $\delta$ 140.1, 134.9, 121.2, 117.1, 78.1, 73.1, 71.2, 66.5, 36.6, 29.5, 26.5, 23.2, 16.5; HRMS $\left(\mathrm{ES}^{+}\right) \mathrm{m} / z$ : $[\mathrm{M}+\mathrm{Na}]^{+}$calcd for $\mathrm{C}_{13} \mathrm{H}_{24} \mathrm{O}_{3} \mathrm{Na}$, 251.1623; found, 251.1631. (R,E)-8-(Allyloxy)-2,6-dimethyloct-6ene-2,3-diol $[(R)-5 \mathbf{b}]$ was prepared under identical conditions but using $\mathrm{AD}-\operatorname{mix}-\beta .[\alpha]_{\mathrm{D}}^{20}+26.1$ ( c 1.0, $\left.\mathrm{CHCl}_{3}\right)$.

(R,E)-3-(5-(Allyloxy)-3-methylpent-3-en-1-yl)-2,2-dimethyloxirane $[(R, E)-5 a] .{ }^{31}$ To a solution of diol $(S)-5 \mathbf{b}(208 \mathrm{mg}, 0.91 \mathrm{mmol}$, 1.0 equiv) in $\mathrm{CH}_{2} \mathrm{Cl}_{2}(1.8 \mathrm{~mL})$ at $0{ }^{\circ} \mathrm{C}$ was added $\mathrm{MsCl}(0.11 \mathrm{~mL}, 1.4$ mmol, 1.5 equiv) followed by pyridine $(0.59 \mathrm{~mL}, 7.3 \mathrm{mmol}, 8.0$ equiv) dropwise. The reaction mixture was allowed to gradually warm to room temperature and was stirred for $20 \mathrm{~h}$, after which the mixture was poured into a suspension of $\mathrm{K}_{2} \mathrm{CO}_{3}(1.9 \mathrm{~g}, 14 \mathrm{mmol}, 15$ equiv $)$ in $\mathrm{MeOH}(9 \mathrm{~mL})$. This suspension was stirred for a further $18 \mathrm{~h}$. The reaction mixture was then concentrated, diluted with $\mathrm{H}_{2} \mathrm{O}(80 \mathrm{~mL})$, and extracted with EtOAc $(3 \times 40 \mathrm{~mL})$. The combined organics were washed with a saturated aqueous $\mathrm{CuSO}_{4}$ solution $(3 \times 50 \mathrm{~mL})$, then brine $(50 \mathrm{~mL})$, dried over $\mathrm{Na}_{2} \mathrm{SO}_{4}$, concentrated, and chromatographed (5-10\% EtOAc in petrol), to give epoxide $(R, E)-5$ a $(171$ $\mathrm{mg}, 0.81 \mathrm{mmol}, 89 \%)$ as a colorless oil. $R_{f} 0.57$ (30\% EtOAc in pentanes); $[\alpha]_{\mathrm{D}}^{22}+4.1\left(\right.$ c 1.0, $\left.\mathrm{CHCl}_{3}\right)$. Data are otherwise identical to the racemic material $(E)-5$ a.

(S,E)-8-(Allyloxy)-2-hydroxy-2,6-dimethyloct-6-en-3-yl Benzoate $[(S)-5 c]$. To a solution of diol $(S)-5 \mathbf{b}(25.0 \mathrm{mg}, 0.11 \mathrm{mmol}, 1.0$ equiv $)$ in pyridine $(1 \mathrm{~mL})$ was added benzoyl chloride $(16 \mu \mathrm{L}, 0.14 \mathrm{mmol}$, 1.3 equiv) and the reaction mixture was stirred at room temperature for $18 \mathrm{~h}$. Then was added additional benzoyl chloride $(33 \mu \mathrm{L}, 0.28$ mmol, 2.6 equiv) and the mixture was stirred for an additional $5 \mathrm{~h}$. The reaction mixture was diluted with EtOAc $(25 \mathrm{~mL})$ and washed with a $1 \mathrm{M}$ aqueous $\mathrm{HCl}$ solution $(3 \times 20 \mathrm{~mL})$ and a saturated aqueous $\mathrm{NaHCO}_{3}$ solution $(3 \times 20 \mathrm{~mL})$. The organics were dried over $\mathrm{Na}_{2} \mathrm{SO}_{4}$, concentrated, and chromatographed on silica gel $(20 \%$ EtOAc in $n$-hexane), to give benzoate $(S)-5 \mathrm{c}(33.0 \mathrm{mg}, 0.11 \mathrm{mmol}$, $99 \%$ ) as a colorless oil. $10.14469 / \mathrm{hpc} / 5744 . R_{f} 0.41$ (40\% EtOAc in $n$-hexane); $[\alpha]_{\mathrm{D}}^{20}-16.7$ (c 1.0, $\mathrm{CHCl}_{3}$ ); IR (ATR, neat) 3600-3250, 3064, $1715 \mathrm{~cm}^{-1} ;{ }^{1} \mathrm{H}$ NMR $\left(400 \mathrm{MHz}, \mathrm{CDCl}_{3}\right): \delta 8.11-8.02(\mathrm{~m}$, $2 \mathrm{H}), 7.63-7.54(\mathrm{~m}, 1 \mathrm{H}), 7.50-7.42(\mathrm{~m}, 2 \mathrm{H}), 5.91(\mathrm{ddt}, J=17.3$, $10.4,5.7 \mathrm{~Hz}, 1 \mathrm{H}), 5.38-5.30(\mathrm{~m}, 1 \mathrm{H}), 5.30-5.22(\mathrm{~m}, 1 \mathrm{H}), 5.20-$ $5.14(\mathrm{~m}, 1 \mathrm{H}), 5.07(\mathrm{dd}, J=9.6,3.3 \mathrm{~Hz}, 1 \mathrm{H}), 4.01-3.86(\mathrm{~m}, 4 \mathrm{H})$, 2.13-2.03 (m, 2H), 1.98-1.74 (m, 3H), $1.65(\mathrm{~s}, 3 \mathrm{H}), 1.27(\mathrm{~s}, 6 \mathrm{H})$; ${ }^{13} \mathrm{C}\left\{{ }^{1} \mathrm{H}\right\}$ NMR $\left(101 \mathrm{MHz}, \mathrm{CDCl}_{3}\right): \delta 166.8,139.4,135.1,133.3$, 130.2, 129.8, 128.6, 121.4, 117.1, 80.4, 72.8, 71.2, 66.6, 36.1, 27.9, 26.7, 25.3, 16.7; HRMS $\left(\mathrm{ES}^{+}\right) \mathrm{m} / z:[\mathrm{M}+\mathrm{Na}]^{+}$calcd for $\mathrm{C}_{20} \mathrm{H}_{28} \mathrm{O}_{4} \mathrm{Na}$, 355.1885; found, 355.1894. HPLC (CHIRALCEL OD; $10 \%$ IPA in $n$-hexane; $0.5 \mathrm{~mL} / \mathrm{min}) t_{\mathrm{R}}=10.1 \mathrm{~min}$ (major), 10.9 min (minor) (96:4). (R,E)-8-(Allyloxy)-2-hydroxy-2,6-dimethyloct-6-en-3-yl benzoate $[(R)-5 \mathbf{c}]$ was prepared under identical conditions from diol $(R)-5 \mathbf{b}$. $[\alpha]_{\mathrm{D}}^{31}+12.4\left(c\right.$ 0.8, $\left.\mathrm{CHCl}_{3}\right)$. HPLC (CHIRALCEL OD; 10\% IPA in $n$-hexane; $0.5 \mathrm{~mL} / \mathrm{min}$ ) $t_{\mathrm{R}}=9.9 \mathrm{~min}$ (minor), $10.6 \mathrm{~min}$ (major) (4:96).

(S,E)-5-(5-(Allyloxy)-3-methylpent-3-en-1-yl)-2,2,4,4-tetramethyl1,3-dioxolane [(S)-5d]. To a solution of diol (S)-5b (1.05 g, 4.59 mmol, 1.0 equiv) in $\mathrm{CH}_{2} \mathrm{Cl}_{2}(9 \mathrm{~mL})$ were added dimethoxypropane $(5.60 \mathrm{~mL}, 45.9 \mathrm{mmol}, 10.0$ equiv) and pyridinium $p$-toluenesulfonate ( $577 \mathrm{mg}, 2.30 \mathrm{mmol}, 0.5$ equiv) at room temperature and the mixture was stirred for $18 \mathrm{~h}$. The reaction mixture was concentrated and loaded directly onto a column of silica gel and chromatographed (5$10 \%$ EtOAc in petrol) to give acetonide $(S)-5 \mathrm{~d}(1.11 \mathrm{~g}, 4.13 \mathrm{mmol}$, $90 \%)$ as a colorless oil. $10.14469 / \mathrm{hpc} / 5745 . R_{f} 0.23$ (5\% EtOAc in petrol); $[\alpha]_{\mathrm{D}}^{24} 2.1$ (c 1.0, $\mathrm{CHCl}_{3}$ ); IR (ATR, neat) $3075,1670 \mathrm{~cm}^{-1}$; ${ }^{1} \mathrm{H}$ NMR $\left(400 \mathrm{MHz}, \mathrm{CDCl}_{3}\right): \delta 5.99-5.86(\mathrm{~m}, 1 \mathrm{H}), 5.44-5.35(\mathrm{~m}$, $1 \mathrm{H}), 5.27$ (br d, $J=17.2 \mathrm{~Hz}, 1 \mathrm{H}), 5.17(\mathrm{br} \mathrm{d}, J=10.4 \mathrm{~Hz}, 1 \mathrm{H}), 3.99$ $(\mathrm{d}, J=6.8 \mathrm{~Hz}, 2 \mathrm{H}), 3.96(\mathrm{~d}, J=5.7 \mathrm{~Hz}, 2 \mathrm{H}), 3.65(\mathrm{dd}, J=9.5,3.3 \mathrm{~Hz}$, $1 \mathrm{H}), 2.34-2.20(\mathrm{~m}, 1 \mathrm{H}), 2.13-2.00(\mathrm{~m}, 1 \mathrm{H}), 1.68(\mathrm{~s}, 3 \mathrm{H}), 1.69-$ $1.58(\mathrm{~m}, 1 \mathrm{H}), 1.56-1.43(\mathrm{~m}, 1 \mathrm{H}), 1.41(\mathrm{~s}, 3 \mathrm{H}), 1.32(\mathrm{~s}, 3 \mathrm{H}), 1.23(\mathrm{~s}$, $3 \mathrm{H}), 1.08(\mathrm{~s}, 3 \mathrm{H}) ;{ }^{13} \mathrm{C}\left\{{ }^{1} \mathrm{H}\right\} \operatorname{NMR}\left(101 \mathrm{MHz}, \mathrm{CDCl}_{3}\right): \delta$ 139.6, 
135.0, 121.1, 117.0, 106.5, 82.9, 80.1, 71.1, 66.5, 36.7, 28.6, 27.5, 26.9, 26.1, 22.9, 16.6; HRMS $\left(\mathrm{EI}^{+}\right) \mathrm{m} / z$ : $[\mathrm{M}]^{\bullet+}$ calcd for $\mathrm{C}_{16} \mathrm{H}_{28} \mathrm{O}_{3}$, 268.2038; found, 268.2050. (R,E)-5-(5-(Allyloxy)-3-methylpent-3-en1-yl)-2,2,4,4-tetramethyl-1,3-dioxolane $[(R)-5 \mathbf{d}]$ was prepared under identical conditions from diol $(R)-5 \mathbf{b}$. $[\alpha]_{\mathrm{D}}^{24}-1.5\left(c 1.0, \mathrm{CHCl}_{3}\right)$.

(S)-2,2,4,4-Tetramethyl-5-(3-methylbut-3-en-1-yl)-1,3-dioxolane [(S)-5f]. To a solution of $(S, E)$-acetonide $(S)-5 \mathbf{d}(100 \mathrm{mg}, 0.37 \mathrm{mmol}$, 1.0 equiv) in $\mathrm{CH}_{2} \mathrm{Cl}_{2}(37 \mathrm{~mL})$ was added ruthenium benzylidene 1 (3.2 $\mathrm{mg}, 0.0037 \mathrm{mmol}, 1 \mathrm{~mol} \%$ ). The mixture was heated to reflux with stirring for $2 \mathrm{~h}$. The reaction mixture was concentrated and loaded directly onto a column of silica gel and chromatographed (5\% EtOAc in petrol) to give alkene $(S)-5 f(57 \mathrm{mg}, 0.28 \mathrm{mmol}, 77 \%)$ as a colorless oil. 10.14469/hpc/5755. $R_{f} 0.53$ (5\% EtOAc in petrol); $[\alpha]_{\mathrm{D}}^{24}-1.4\left(c 1.0, \mathrm{CHCl}_{3}\right)$; IR (ATR, neat) $3071,1648 \mathrm{~cm}^{-1} ;{ }^{1} \mathrm{H}$ NMR (400 MHz, CDCl): $\delta 4.74(\mathrm{~s}, 1 \mathrm{H}), 4.71(\mathrm{~s}, 1 \mathrm{H}), 3.68(\mathrm{dd}, J=$ 9.4, $3.5 \mathrm{~Hz}, 1 \mathrm{H}), 2.31-2.17(\mathrm{~m}, 1 \mathrm{H}), 2.12-2.00(\mathrm{~m}, 1 \mathrm{H}), 1.74(\mathrm{~s}$, $3 \mathrm{H}), 1.71-1.60(\mathrm{~m}, 1 \mathrm{H}), 1.57-1.46(\mathrm{~m}, 1 \mathrm{H}), 1.42(\mathrm{~s}, 3 \mathrm{H}), 1.32(\mathrm{~s}$, $3 \mathrm{H}), 1.24(\mathrm{~s}, 3 \mathrm{H}), 1.10(\mathrm{~s}, 3 \mathrm{H}) ;{ }^{13} \mathrm{C}\left\{{ }^{1} \mathrm{H}\right\} \mathrm{NMR}\left(101 \mathrm{MHz}, \mathrm{CDCl}_{3}\right)$ : $\delta 145.2,110.1,106.5,82.8,80.1,34.9,28.6,27.4,26.9,26.0,22.9$, 22.6; HRMS $\left(\mathrm{EI}^{+}\right) \mathrm{m} / z$ : $[\mathrm{M}]^{\bullet+}$ calcd for $\mathrm{C}_{12} \mathrm{H}_{22} \mathrm{O}_{2}, 198.1620$; found, 198.1612 .

$(S, E)-5-(5-(A l l y l o x y)-3-m e t h y l p e n t-3-e n-1-y l)-4,4-d i m e t h y l-2-$ phenyl-1,3,2-dioxaborolane [(S)-5g]. Phenylboronic acid (315 mg, $2.63 \mathrm{mmol}, 1.05$ equiv) was added to a solution of diol $(S)-5 \mathbf{b}(570$ $\mathrm{mg}, 2.5 \mathrm{mmol}, 1$ equiv) in EtOAc $(10 \mathrm{~mL})$. After $90 \mathrm{~min}$, the reaction mixture was concentrated, passed through a silica plug (15\% EtOAc in petrol; $150 \mathrm{~mL})$, and evaporated to give boronate $(S)-5 \mathrm{~g}(775 \mathrm{mg}$, $2.50 \mathrm{mmol}, 99 \%)$ as a pale beige viscous oil. $10.14469 / \mathrm{hpc} / 6317$. $[\alpha]_{\mathrm{D}}^{25}-17.1\left(c 1.0, \mathrm{CHCl}_{3}\right.$ ); IR (ATR, neat) $3075 \mathrm{~cm}^{-1} ;{ }^{1} \mathrm{H}$ NMR $\left(400 \mathrm{MHz}, \mathrm{CDCl}_{3}\right): \delta 7.82(\mathrm{~d}, J=7.1 \mathrm{~Hz}, 2 \mathrm{H}), 7.51-7.42(\mathrm{~m}, 1 \mathrm{H})$, $7.41-7.33(\mathrm{~m}, 2 \mathrm{H}), 6.00-5.89(\mathrm{~m}, 1 \mathrm{H}), 5.46(\mathrm{t}, J=6.6 \mathrm{~Hz}, 1 \mathrm{H})$, $5.28(\mathrm{~d}, J=17.2 \mathrm{~Hz}, 1 \mathrm{H}), 5.19(\mathrm{~d}, J=10.3 \mathrm{~Hz}, 1 \mathrm{H}), 4.06(\mathrm{dd}, J=$ $10.3,3.4 \mathrm{~Hz}, 1 \mathrm{H}), 4.02(\mathrm{~d}, J=6.7 \mathrm{~Hz}, 2 \mathrm{H}), 3.99(\mathrm{~d}, J=5.7 \mathrm{~Hz}, 2 \mathrm{H})$, 2.48-2.38 (m, 1H), 2.23-2.12 (m, 1H), 1.81-1.69 (m, $1 \mathrm{H}), 1.73(\mathrm{~s}$, $3 \mathrm{H}), 1.68-1.59(\mathrm{~m}, 1 \mathrm{H}), 1.44(\mathrm{~s}, 3 \mathrm{H}), 1.29(\mathrm{~s}, 3 \mathrm{H}) ;{ }^{13} \mathrm{C}\left\{{ }^{1} \mathrm{H}\right\} \mathrm{NMR}$ $\left(101 \mathrm{MHz}, \mathrm{CDCl}_{3}\right): \delta$ 139.6, 135.0, 134.8, 131.3, 127.8, 121.2, 117.1,

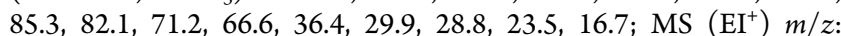
$[\mathrm{M}]^{\bullet+}$ calcd for $\mathrm{C}_{19} \mathrm{H}_{27}{ }^{11} \mathrm{BO}_{3}, 314.2053$; found, 314.2062. HPLC (CHIRALCEL OD-H, 1\% IPA in $n$-hexane; $1.0 \mathrm{~mL} / \mathrm{min}) t_{\mathrm{R}}=8.5$ min (major), 10.8 min (minor) (95:5). (R,E)-5-(5-(Allyloxy)-3methylpent-3-en-1-yl)-4,4-dimethyl-2-phenyl-1,3,2-dioxaborolane $[(R)-5 \mathbf{g}]$ was prepared under identical conditions from diol $(R)-5 \mathbf{b}$. $[\alpha]_{\mathrm{D}}^{25}+15.8\left(c 1.0, \mathrm{CHCl}_{3}\right)$. HPLC (CHIRALCEL OD-H, 1\% IPA in $n$-hexane; $1.0 \mathrm{~mL} / \mathrm{min}$ ) $t_{\mathrm{R}}=8.5 \mathrm{~min}$ (minor), $10.8 \mathrm{~min}$ (major) (3:97).

General Procedure for Preparation of Trisubstituted Alkenes 7 via Wittig Olefination of Aldehydes. Using a modified procedure of Pfaltz, ${ }^{20}$ to a suspension of $\left(\mathrm{CH}_{3}\right)_{2} \mathrm{CHPPh}_{3} \mathrm{I} 6$ (7.8 g, 18 mmol, 1.8 equiv) in THF $(20 \mathrm{~mL})$ was added ${ }^{n} \mathrm{BuLi}(11.25 \mathrm{~mL}, 1.6$ $\mathrm{M}$ in hexanes, $18 \mathrm{mmol}, 1.8$ equiv) dropwise at $0{ }^{\circ} \mathrm{C}$. After $30 \mathrm{~min}$, the required aldehyde (1.0 equiv) was added dropwise and the mixture was stirred for $18 \mathrm{~h}$. The mixture was quenched by addition of $\mathrm{H}_{2} \mathrm{O}(15 \mathrm{~mL})$ and the THF was removed by concentration. The mixture was diluted with $\mathrm{Et}_{2} \mathrm{O}(100 \mathrm{~mL})$ and filtered through a pad of Celite. The organics were washed with $\mathrm{H}_{2} \mathrm{O}(3 \times 20 \mathrm{~mL})$, dried over $\mathrm{Na}_{2} \mathrm{SO}_{4}$, and concentrated. The crude material was purified by DCVC, eluting with pentanes, to give the desired trisubstituted alkene.

(4-Methylpent-3-en-1-yl)benzene (7a). ${ }^{32}$ Following the general procedure for the formation of trisubstituted alkenes using hydrocinnamaldehyde $(1.32 \mathrm{~mL}, 10.0 \mathrm{mmol}, 1.0$ equiv) gave alkene $7 \mathrm{a}$ $(0.99 \mathrm{~g}, 6.1 \mathrm{mmol}, 61 \%)$ as a colorless oil. $10.14469 / \mathrm{hpc} / 5759 . R_{f}$ 0.44 (pentanes); IR (ATR, neat) $3027 \mathrm{~cm}^{-1}$; ${ }^{1} \mathrm{H}$ NMR (400 MHz, $\left.\mathrm{CDCl}_{3}\right): \delta 7.31-7.22(\mathrm{~m}, 2 \mathrm{H}), 7.22-7.12(\mathrm{~m}, 3 \mathrm{H}), 5.24-5.09(\mathrm{~m}$, $1 \mathrm{H}), 2.68-2.58(\mathrm{~m}, 2 \mathrm{H}), 2.29(\mathrm{q}, J=7.7 \mathrm{~Hz}, 2 \mathrm{H}), 1.68(\mathrm{~s}, 3 \mathrm{H}), 1.56$ $(\mathrm{s}, 3 \mathrm{H}) ;{ }^{13} \mathrm{C}\left\{{ }^{1} \mathrm{H}\right\}$ NMR $\left(101 \mathrm{MHz}, \mathrm{CDCl}_{3}\right): \delta 142.5,132.3,128.6$, $128.3,125.8,123.9,36.3,30.2,25.8,17.8$; HRMS $\left(\mathrm{CI}^{+}\right) \mathrm{m} / z:[\mathrm{M}+$ $\mathrm{H}]^{+}$calcd for $\mathrm{C}_{12} \mathrm{H}_{17}, 161.1330$; found, 161.1325.

1-Methoxy-4-(4-methylpent-3-en-1-yl)benzene (7c). ${ }^{36}$ To a solution of $p$-methoxybenzyl chloride $(2.26 \mathrm{~mL}, 16.6 \mathrm{mmol}, 1.0$ equiv) in THF $(10 \mathrm{~mL})$ was added dropwise over $20 \mathrm{~min}$ to magnesium turnings ( $0.478 \mathrm{~g}, 20.0 \mathrm{mmol}, 1.2$ equiv) in THF $(10 \mathrm{~mL})$ and at $0{ }^{\circ} \mathrm{C}$. The reaction was warmed to room temperature and the mixture was stirred for $3 \mathrm{~h}$. To the mixture was added dropwise a solution of tetrakis(triphenylphosphine)palladium(0) (0.226 g, 0.196 mmol, $1.5 \mathrm{~mol} \%)$ and 1-bromo-3-methylbut-2-ene (2.4 mL, 20.1 mmol, 1.0 equiv) in THF $(10 \mathrm{~mL})$ at $-78^{\circ} \mathrm{C}$. The reaction mixture turned to green immediately and was stirred for an additional $3 \mathrm{~h}$ before warming to room temperature. The reaction mixture was stirred for $16 \mathrm{~h}$ and quenched with ice water $(20 \mathrm{~mL})$, then extracted with $\mathrm{Et}_{2} \mathrm{O}(2 \times 20 \mathrm{~mL})$. The organics were washed with water $(20$ $\mathrm{mL})$, brine $(20 \mathrm{~mL})$, dried over $\mathrm{Na}_{2} \mathrm{SO}_{4}$, concentrated, and chromatographed (20\% EtOAc in $n$-hexane) to give alkene $7 \mathrm{c}$ (2.29 g, $12.3 \mathrm{mmol}, 70 \%$, containing $5 \%$ of inseparable $p$-methoxytoluene). $10.14469 / \mathrm{hpc} / 5761 . R_{f} 0.20$ (10\% EtOAc in $n$-hexane); ${ }^{1} \mathrm{H}$ NMR $\left(400 \mathrm{MHz}, \mathrm{CDCl}_{3}\right): \delta 7.12(\mathrm{~d}, J=8.6 \mathrm{~Hz}, 2 \mathrm{H}), 6.84(\mathrm{~d}, J=8.6 \mathrm{~Hz}$, $2 \mathrm{H}), 5.18(\mathrm{t}, J=7.1,1 \mathrm{H}), 3.80(\mathrm{~s}, 3 \mathrm{H}), 2.58(\mathrm{t}, J=7.8 \mathrm{~Hz}, 2 \mathrm{H}), 2.27$ (app. q, $J=7.8 \mathrm{~Hz}, 2 \mathrm{H}), 1.70(\mathrm{~s}, 3 \mathrm{H}), 1.58(\mathrm{~s}, 3 \mathrm{H}) ;{ }^{13} \mathrm{C}\left\{{ }^{1} \mathrm{H}\right\} \mathrm{NMR}$ $\left(101 \mathrm{MHz}, \mathrm{CDCl}_{3}\right): \delta 157.7,134.6,132.1,129.3,123.8,113.6,55.3$, 35.2, 30.3, 25.7, 17.7; HRMS $\left(\mathrm{CI}^{+}\right) \mathrm{m} / z:[\mathrm{M}-\mathrm{H}]^{+}$calcd for $\mathrm{C}_{13} \mathrm{H}_{17} \mathrm{O}$, 189.1274; found, 189.1272 .

1-Methoxy-3-(4-methylpent-3-en-1-yl)benzene (7d). Following the general procedure for the formation of trisubstituted alkenes using $\left(\mathrm{CH}_{3}\right)_{2} \mathrm{CHPPh}_{3} \mathrm{I} 6$ (3.8 g, $8.8 \mathrm{mmol}, 1.8$ equiv), THF $(10 \mathrm{~mL})$, ${ }^{n} \mathrm{BuLi}(3.5 \mathrm{~mL}, 2.5 \mathrm{M}$ in hexanes, $8.8 \mathrm{mmol}, 1.8$ equiv), and 3-(3methoxyphenyl)propanal ${ }^{33}(0.8 \mathrm{~g}, 4.9 \mathrm{mmol}, 1.0$ equiv) gave alkene $7 \mathrm{~d}(0.55 \mathrm{~g}, 59 \%)$ as a colorless oil. $10.14469 / \mathrm{hpc} / 6318 . R_{f} 0.85(10 \%$ EtOAc in petrol); ${ }^{1} \mathrm{H}$ NMR $\left(400 \mathrm{MHz}, \mathrm{CDCl}_{3}\right): \delta 7.22-7.15(\mathrm{~m}$, $1 \mathrm{H}), 6.82-6.77(\mathrm{~m}, 1 \mathrm{H}), 6.76-6.72(\mathrm{~m}, 2 \mathrm{H}), 5.23-5.12(\mathrm{~m}, 1 \mathrm{H})$, $3.80(\mathrm{~s}, 3 \mathrm{H}), 2.71-2.57$ (m, 2H), 2.29 (app. q, $J=7.6 \mathrm{~Hz}, 2 \mathrm{H}), 1.69$ $(\mathrm{d}, J=1.3 \mathrm{~Hz}, 3 \mathrm{H}), 1.58(\mathrm{~s}, 3 \mathrm{H}) ;{ }^{13} \mathrm{C}\left\{{ }^{1} \mathrm{H}\right\}$ NMR $\left(101 \mathrm{MHz}, \mathrm{CDCl}_{3}\right)$ : $\delta 159.7,144.2,132.2,129.3,123.8,121.0,114.3,111.1,55.2,36.3$, 30.0, 25.8, 17.8; HRMS $\left(\mathrm{CI}^{+}\right) \mathrm{m} / z:[\mathrm{M}+\mathrm{H}]^{+}$calcd for $\mathrm{C}_{13} \mathrm{H}_{19} \mathrm{O}$, 191.1430; found, 191.1430.

1-Methoxy-2-(4-methylpent-3-en-1-yl)benzene (7e). ${ }^{34}$ Following the general procedure for the formation of trisubstituted alkenes using $\left(\mathrm{CH}_{3}\right)_{2} \mathrm{CHPPh}_{3} \mathrm{I} 6$ (3.9 g, $9.0 \mathrm{mmol}, 1.8$ equiv), THF $(10 \mathrm{~mL}),{ }^{n} \mathrm{BuLi}$ (3.6 mL, 2.5 $\mathrm{M}$ in hexanes, $9.0 \mathrm{mmol}, 1.8$ equiv), and 3-(2methoxyphenyl)propanal ${ }^{35}(0.82 \mathrm{~g}, 5.0 \mathrm{mmol}, 1.0$ equiv) gave alkene $7 \mathrm{e}(0.73 \mathrm{~g}, 3.8 \mathrm{mmol}, 76 \%)$ as a colorless oil. $10.14469 / \mathrm{hpc} / 6319 . R_{f}$ 0.20 (5\% EtOAc in petrol); ${ }^{1} \mathrm{H}$ NMR $\left(400 \mathrm{MHz}, \mathrm{CDCl}_{3}\right): \delta 7.22-$ $7.10(\mathrm{~m}, 2 \mathrm{H}), 6.92-6.81(\mathrm{~m}, 2 \mathrm{H}), 5.21(\mathrm{~m}, 1 \mathrm{H}), 3.83(\mathrm{~s}, 3 \mathrm{H}), 2.68-$ $2.57(\mathrm{~m}, 2 \mathrm{H}), 2.31-2.20(\mathrm{~m}, 2 \mathrm{H}), 1.69(\mathrm{~d}, J=1.4 \mathrm{~Hz}, 3 \mathrm{H}), 1.58(\mathrm{~d}, J$ $=1.4 \mathrm{~Hz}, 3 \mathrm{H}) ;{ }^{13} \mathrm{C}\left\{{ }^{1} \mathrm{H}\right\}$ NMR (101 MHz, $\left.\mathrm{CDCl}_{3}\right): \delta 157.5,131.8$, 130.8, 129.8, 126.9, 124.3, 120.3, 110.2, 55.3, 30.6, 28.3, 25.7, 17.6; MS $\left(\mathrm{CI}^{+}\right) \mathrm{m} / z:[\mathrm{M}+\mathrm{H}]^{+}$calcd for $\mathrm{C}_{13} \mathrm{H}_{19} \mathrm{O}, 191.1430$; found, 191.1431.

1-Methyl-4-(4-methylpent-3-en-1-yl)benzene (7f). ${ }^{36}$ Following the general procedure for the formation of trisubstituted alkenes using $\left(\mathrm{CH}_{3}\right)_{2} \mathrm{CHPPh}_{3} \mathrm{I} 6$ (2.5 g, $5.8 \mathrm{mmol}, 1.2$ equiv), THF $(10 \mathrm{~mL})$, ${ }^{n} \mathrm{BuLi}(2.3 \mathrm{~mL}, 2.5 \mathrm{M}$ in hexanes, $5.8 \mathrm{mmol}, 1.2$ equiv), and 3-(4methylphenyl)propanal ${ }^{37}(0.72 \mathrm{~g}, 4.9 \mathrm{mmol}, 1.0$ equiv) gave alkene $7 \mathrm{f}$ $(0.51 \mathrm{~g}, 2.9 \mathrm{mmol}, 60 \%)$ as a colorless oil. $10.14469 / \mathrm{hpc} / 6320 . R_{f}$ 0.93 (10\% EtOAc in petrol); IR (ATR, neat) $3042 \mathrm{~cm}^{-1}$; ${ }^{1} \mathrm{H}$ NMR $\left(400 \mathrm{MHz}, \mathrm{CDCl}_{3}\right): \delta 7.10(\mathrm{~s}, 4 \mathrm{H}), 5.26-5.12(\mathrm{~m}, 1 \mathrm{H}), 2.66-2.56$ (m, 2H), 2.33 (s, 3H), 2.29 (app. q, $J=7.7 \mathrm{~Hz}, 2 \mathrm{H}), 1.70(\mathrm{~s}, 3 \mathrm{H})$, $1.59(\mathrm{~s}, 3 \mathrm{H}) ;{ }^{13} \mathrm{C}\left\{{ }^{1} \mathrm{H}\right\}$ NMR $\left(101 \mathrm{MHz} \mathrm{CDCl}_{3}\right): \delta 139.5,135.2$, 132.2, 129.1, 128.4, 124.0, 35.8, 30.3, 25.8, 21.2, 17.8; HRMS $\left(\mathrm{EI}^{+}\right)$ $\mathrm{m} / z:[\mathrm{M}]^{\bullet+}$ calcd for $\mathrm{C}_{13} \mathrm{H}_{18}, 174.1409$; found, 174.1411 .

1-Chloro-4-(4-methylpent-3-en-1-yl)benzene (7g). ${ }^{38}$ Following the general procedure for the formation of trisubstituted alkenes using $\left(\mathrm{CH}_{3}\right)_{2} \mathrm{CHPPh}_{3} \mathrm{I} 6$ (3.9 g, $9.0 \mathrm{mmol}, 1.8$ equiv), THF $(10 \mathrm{~mL})$, ${ }^{n} \mathrm{BuLi}(3.6 \mathrm{~mL}, 2.5 \mathrm{M}$ in hexanes, $9.0 \mathrm{mmol}, 1.8$ equiv), and 3-(4chlorophenyl)propanal $^{39}(843 \mathrm{mg}, 5.0 \mathrm{mmol}, 1.0$ equiv) gave alkene $7 \mathrm{~g}$ (828 mg, $4.3 \mathrm{mmol}, 85 \%)$ as a colorless oil. $10.14469 / \mathrm{hpc} / 6321$. $R_{f} 0.30$ (5\% EtOAc in petrol); ${ }^{1} \mathrm{H}$ NMR $\left(400 \mathrm{MHz} \mathrm{CDCl}_{3}\right): \delta 7.26-$ $7.21(\mathrm{~m}, 2 \mathrm{H}), 7.13-7.08(\mathrm{~m}, 2 \mathrm{H}), 5.13(\mathrm{~m}, 1 \mathrm{H}), 2.63-2.56(\mathrm{~m}, 2 \mathrm{H})$, $2.31-2.22(\mathrm{~m}, 2 \mathrm{H}), 1.68(\mathrm{~d}, J=1.4 \mathrm{~Hz}, 3 \mathrm{H}), 1.54(\mathrm{~d}, J=1.4 \mathrm{~Hz}$, $3 \mathrm{H}) ;{ }^{13} \mathrm{C}\left\{{ }^{1} \mathrm{H}\right\}$ NMR (101 MHz, $\left.\mathrm{CDCl}_{3}\right): \delta 140.8,132.5,131.4$, 
129.8, 128.3, 123.3, 35.4, 29.9, 25.7, 17.7; $\mathrm{MS}\left(\mathrm{CI}^{+}\right) m / z:[\mathrm{M}-\mathrm{H}]^{+}$ calcd for $\mathrm{C}_{12} \mathrm{H}_{14}{ }^{35} \mathrm{Cl}$, 193.0779; found, 193.0777.

2-Methylnon-2-ene (7h). ${ }^{40}$ Following the general procedure for the formation of trisubstituted alkenes using heptanal $(1.40 \mathrm{~mL}, 10.0$ mmol, 1.0 equiv) gave alkene $7 \mathrm{~h}(0.59 \mathrm{~g}, 4.2 \mathrm{mmol}, 42 \%)$ as a colorless oil. 10.14469/hpc/5756. $R_{f} 0.94$ (pentanes); ${ }^{1} \mathrm{H}$ NMR (400 $\left.\mathrm{MHz}, \mathrm{CDCl}_{3}\right): \delta 5.24-5.01(\mathrm{~m}, 1 \mathrm{H}), 2.03-1.88(\mathrm{~m}, 2 \mathrm{H}), 1.69(\mathrm{~d}, J=$ $1.4 \mathrm{~Hz}, 3 \mathrm{H}), 1.60(\mathrm{~s}, 3 \mathrm{H}), 1.36-1.23(\mathrm{~m}, 8 \mathrm{H}), 0.89(\mathrm{t}, J=6.7 \mathrm{~Hz}$, $3 \mathrm{H}) ;{ }^{13} \mathrm{C}\left\{{ }^{1} \mathrm{H}\right\} \mathrm{NMR}\left(101 \mathrm{MHz}, \mathrm{CDCl}_{3}\right): \delta 131.2,125.1,32.0,30.0$, 29.2, 28.2, 25.9, 22.8, 17.8, 14.3; HRMS $\left(\mathrm{EI}^{+}\right) \mathrm{m} / z$ : $[\mathrm{M}]^{\bullet+}$ calcd for $\mathrm{C}_{10} \mathrm{H}_{20}, 140.1560$; found, 140.1554 .

4-Ethyl-2-methyloct-2-ene (7i). Following the general procedure for the formation of trisubstituted alkenes using 2-ethylhexanal (1.56 $\mathrm{mL}, 10.0 \mathrm{mmol}, 1.0$ equiv) gave alkene $7 \mathbf{i}(0.87 \mathrm{~g}, 5.7 \mathrm{mmol}, 57 \%)$ as a colorless oil. $10.14469 / \mathrm{hpc} / 5760 . R_{f} 0.92$ (pentanes); ${ }^{1} \mathrm{H}$ NMR $\left(400 \mathrm{MHz}, \mathrm{CDCl}_{3}\right): \delta 4.79$ (br d, $\left.J=9.8 \mathrm{~Hz}, 1 \mathrm{H}\right), 2.13-2.01(\mathrm{~m}$, $1 \mathrm{H}), 1.71(\mathrm{~d}, J=1.4 \mathrm{~Hz}, 3 \mathrm{H}), 1.60(\mathrm{~d}, J=1.3 \mathrm{~Hz}, 3 \mathrm{H}), 1.45-1.06$ $(\mathrm{m}, 8 \mathrm{H}), 0.87(\mathrm{t}, J=7.1 \mathrm{~Hz}, 3 \mathrm{H}), 0.81(\mathrm{t}, J=7.4 \mathrm{~Hz}, 3 \mathrm{H}) ;{ }^{13} \mathrm{C}\left\{{ }^{1} \mathrm{H}\right\}$ NMR (101 MHz, $\left.\mathrm{CDCl}_{3}\right): \delta 130.8,130.4,39.8,35.8,29.8,29.0,26.0$, 23.1, 18.4, 14.3, 11.9; HRMS $\left(\mathrm{EI}^{+}\right) \mathrm{m} / z$ : $[\mathrm{M}]^{\bullet+}$ calcd for $\mathrm{C}_{11} \mathrm{H}_{22}$, 154.1721; found, 154.1716 .

(2-Methylprop-1-en-1-yl)benzene (7j). ${ }^{20}$ Following the general procedure for the formation of trisubstituted alkenes using benzaldehyde $(1.02 \mathrm{~mL}, 10.0 \mathrm{mmol}, 1.0$ equiv) gave alkene $7 \mathbf{j}(1.30$ g, $9.8 \mathrm{mmol}, 98 \%)$ as a colorless oil. $10.14469 / \mathrm{hpc} / 5757 . R_{f} 0.58$ (pentanes); IR (ATR, neat) 3021, $1657 \mathrm{~cm}^{-1}$; ${ }^{1} \mathrm{H}$ NMR (400 MHz, $\left.\mathrm{CDCl}_{3}\right): \delta 7.37-7.28(\mathrm{~m}, 2 \mathrm{H}), 7.28-7.15(\mathrm{~m}, 3 \mathrm{H}), 6.29(\mathrm{~s}, 1 \mathrm{H}), 1.93$ $(\mathrm{s}, 3 \mathrm{H}), 1.88(\mathrm{~s}, 3 \mathrm{H}) ;{ }^{13} \mathrm{C}\left\{{ }^{1} \mathrm{H}\right\}$ NMR $\left(101 \mathrm{MHz}, \mathrm{CDCl}_{3}\right): \delta 138.9$, 135.6, 128.9, 128.2, 125.9, 125.3, 27.0, 19.6; HRMS $\left(\mathrm{EI}^{+}\right) \mathrm{m} / z:[\mathrm{M}]^{\bullet+}$ calcd for $\mathrm{C}_{10} \mathrm{H}_{12}, 132.0934$; found, 132.0933 .

(3-Methylbut-2-en-1-yl)benzene (7k). ${ }^{41}$ Following the general procedure for the formation of trisubstituted alkenes using phenylacetaldehyde ( $1.17 \mathrm{~mL}, 10.0 \mathrm{mmol}, 1.0$ equiv) gave alkene $7 \mathbf{k}(0.40 \mathrm{~g}$, $2.8 \mathrm{mmol}, 28 \%)$ as a colorless oil. $10.14469 / \mathrm{hpc} / 5758 . R_{f} 0.63$ (pentanes); IR (ATR, neat) $3027 \mathrm{~cm}^{-1}$; ${ }^{1} \mathrm{H}$ NMR $(400 \mathrm{MHz}$, $\left.\mathrm{CDCl}_{3}\right): \delta 7.33-7.23(\mathrm{~m}, 2 \mathrm{H}), 7.22-7.13(\mathrm{~m}, 3 \mathrm{H}), 5.40-5.27(\mathrm{~m}$, $1 \mathrm{H}), 3.35(\mathrm{~d}, J=7.4 \mathrm{~Hz}, 2 \mathrm{H}), 1.75(\mathrm{~d}, J=1.4 \mathrm{~Hz}, 3 \mathrm{H}), 1.72(\mathrm{~s}, 3 \mathrm{H})$; ${ }^{13} \mathrm{C}\left\{{ }^{1} \mathrm{H}\right\}$ NMR $\left(101 \mathrm{MHz}, \mathrm{CDCl}_{3}\right): \delta 141.9,132.6,128.5,128.4$, 125.8, 123.3, 34.5, 25.9, 17.9; HRMS $\left(\mathrm{EI}^{+}\right) \mathrm{m} / z:[\mathrm{M}]^{\bullet+}$ calcd for $\mathrm{C}_{11} \mathrm{H}_{14}, 146.1090$; found, 146.1084.

General Procedure for Preparation of Trisubstituted Alkenes 8 via Relay Cross-Metathesis. To a neat mixture of relay alkene 5 ( $0.25 \mathrm{mmol}, 1.0$ equiv) and alkene $7(1.25 \mathrm{mmol}, 5.0$ equiv) was added ruthenium benzylidene $1(21 \mathrm{mg}, 0.025 \mathrm{mmol}, 10$ mol \%). The mixture was heated to $50{ }^{\circ} \mathrm{C}$ using an oil bath for $1 \mathrm{~h}$ under a strong positive pressure of $\mathrm{N}_{2}(\mathrm{~g})$ via a needle in/out to aid the removal of volatiles. The resulting mixture was loaded directly onto a column of silica gel and chromatographed.

2,2-Dimethyl-3-(3-methyl-6-phenylhex-3-en-1-yl)oxirane $(8 a)^{2 a}$ Following the general procedure for relay cross-metathesis using epoxy allyl ether $(E)-5 \mathbf{a}(53 \mathrm{mg})$ and alkene $7 \mathbf{a}(200 \mathrm{mg})$ gave trisubstituted alkene $8 \mathrm{a}(51 \mathrm{mg}, 0.21 \mathrm{mmol}, 84 \%$ from $(E)-5 \mathrm{a} ; 42 \mathrm{mg}$, $0.17 \mathrm{mmol}, 69 \%$ from $(Z)-5 \mathrm{a})$ as a colorless oil and a mixture of $E / Z$ geometrical isomers $[E / Z=73: 27$ (from $(E)-5 a$ ) or 79:21 (from $(Z)$ 5a) ]. $10.14469 / \mathrm{hpc} / 5763 . R_{f} 0.50$ (10\% EtOAc in pentanes); IR (ATR, neat) $3026 \mathrm{~cm}^{-1}$; ${ }^{1} \mathrm{H}$ NMR $\left(400 \mathrm{MHz}, \mathrm{CDCl}_{3}\right): \delta 7.30-7.22$ $(\mathrm{m}, 2 \mathrm{H}), 7.20-7.12(\mathrm{~m}, 3 \mathrm{H}), 5.25-5.18(\mathrm{~m}, 1 \mathrm{H}), 2.72-2.59(\mathrm{~m}$, $3 \mathrm{H}), 2.37-2.26(\mathrm{~m}, 2 \mathrm{H}), 2.20-2.01(\mathrm{~m}, 2 \mathrm{H}), 1.71-1.52(\mathrm{~m}, 5 \mathrm{H})$, 1.28 (s, 3H), 1.24 (s, E-8a, 2.19H), 1.24 (s, Z-8a, 0.81H). The $E / Z$ ratio was determined by integration of the resonances at $\delta 1.24(4)$ (major) and 1.23(9) (minor) ppm; ${ }^{13} \mathrm{C}\left\{{ }^{1} \mathrm{H}\right\}$ NMR (101 MHz, $\left.\mathrm{CDCl}_{3}\right): \delta 142.4,142.3,135.0,135.0,128.6,128.4,128.4,125.9$, $125.9,125.2,124.4,64.3,64.2,58.5,58.5,36.5,36.4,36.2,30.1,30.0$, $28.7,27.6,27.5,25.1,25.1,23.5,18.9,18.9,16.1$. The E-isomer was identified as the major isomer on the basis of a characteristic shielded methyl resonance ${ }^{23}$ at 16.1 versus $23.5 \mathrm{ppm}$ for the minor $Z$-isomer; HRMS $\left(\mathrm{CI}^{+}\right) \mathrm{m} / z:[\mathrm{M}+\mathrm{H}]^{+}$calcd for $\mathrm{C}_{17} \mathrm{H}_{25} \mathrm{O}$, 245.1905; found, 245.1900.

((E)-6-(((E)-3,7-Dimethylocta-2,6-dien-1-yl)oxy)hex-4-en-1-yl)benzene (9a) and 2,2-Dimethyl-3-((3E)-3-methyl-5-(prop-1-en-1- yloxy)pent-3-en-1-yl)oxirane (10a). Following the general procedure for relay cross-metathesis using epoxy allyl ether $(E)-5 a(53 \mathrm{mg})$ and alkene $7 \mathbf{b}(165 \mathrm{mg})$, with regular addition of $7 \mathbf{b}$ at $10 \mathrm{~min}$ intervals to keep the reaction volume constant throughout the whole reaction process, gave first 10a (14.3 $\mathrm{mg}, 0.07 \mathrm{mmol}, 27 \%)$ as a colorless oil and a mixture of $E / Z$ geometrical isomers $[E / Z=49: 51]$, and second 9a $(29.0 \mathrm{mg}, 0.09 \mathrm{mmol}, 37 \%)$ as a colorless oil. 9a: $10.14469 / \mathrm{hpc} /$ 6466. $R_{f} 0.30$ (10\% EtOAc in pentanes); IR (ATR, neat) 3023, 1670 $\mathrm{cm}^{-1}$; ${ }^{1} \mathrm{H}$ NMR $\left(400 \mathrm{MHz}, \mathrm{CDCl}_{3}\right): \delta 7.31-7.26(\mathrm{~m}, 2 \mathrm{H}), 7.21-$ $7.15(\mathrm{~m}, 3 \mathrm{H}), 5.80-5.70(\mathrm{~m}, 1 \mathrm{H}), 5.66-5.56(\mathrm{~m}, 1 \mathrm{H}), 5.42-5.35$ (m, $1 \mathrm{H}), 3.95(\mathrm{~d}, J=6.8,2 \mathrm{H}), 3.91(\mathrm{dd}, J=6.0,0.9 \mathrm{~Hz}, 2 \mathrm{H}), 2.71$ $(\mathrm{m}, 3 \mathrm{H}), 2.38(\mathrm{~m}, 2 \mathrm{H}), 2.27-2.07(\mathrm{~m}, 2 \mathrm{H}), 1.71-1.62(\mathrm{~m}, 5 \mathrm{H}), 1.30$ $(\mathrm{s}, 3 \mathrm{H}), 1.26(\mathrm{~s}, 3 \mathrm{H}) ;{ }^{13} \mathrm{C}\left\{{ }^{1} \mathrm{H}\right\}$ NMR $\left(101 \mathrm{MHz}, \mathrm{CDCl}_{3}\right): \delta 141.9$, 139.1, 133.7, 128.4, 128.3, 127.1, 125.8, 121.5, 70.8, 66.3, 64.0, 58.4, 36.2, 35.5, 34.1, 27.2, 24.9, 18.7, 16.5. HRMS $\left(\mathrm{CI}^{+}\right) \mathrm{m} / z:[\mathrm{M}+\mathrm{H}]^{+}$ calcd for $\mathrm{C}_{21} \mathrm{H}_{31} \mathrm{O}_{2}$, 315.2319; found, 315.2323. 10a: $10.14469 / \mathrm{hpc} /$ 6469. $R_{f} 0.60$ (10\% EtOAc in pentanes); IR (ATR, neat) $1662 \mathrm{~cm}^{-1}$; ${ }^{1} \mathrm{H}$ NMR $\left(400 \mathrm{MHz}, \mathrm{CDCl}_{3}\right): \delta 6.22(\mathrm{dq}, J=12.5,1.6 \mathrm{~Hz}, 0.51 \mathrm{H}$, (E)-9a), 5.96 (dq, $J=6.3,1.7 \mathrm{~Hz}, 0.49 \mathrm{H},(Z)-9 \mathrm{a}), 5.41(\mathrm{~m}, 1 \mathrm{H}), 4.79$ $(\mathrm{dq}, J=13.3,6.7 \mathrm{~Hz}, 0.51 \mathrm{H},(E)-9 \mathrm{a}), 4.38$ (appr. qu., $J=6.8 \mathrm{~Hz}$, $0.49 \mathrm{H},(Z)-9 \mathrm{a}), 4.26(\mathrm{~d}, J=6.7 \mathrm{~Hz}, 1.02 \mathrm{H},(E)-9 \mathrm{a}), 4.18(\mathrm{~d}, J=6.7$ $\mathrm{Hz}, 0.98 \mathrm{H},(\mathrm{Z})-9 \mathrm{a}), 2.70(\mathrm{t}, J=6.3 \mathrm{~Hz}, 1 \mathrm{H}), 2.28-2.07(\mathrm{~m}, 2 \mathrm{H})$, $1.73-1.60(\mathrm{~m}, 5 \mathrm{H}), 1.57$ (dd, $J=6.8,1.7 \mathrm{~Hz}, 1.53 \mathrm{H},(E)-9 \mathrm{a}), 1.55$ $(\mathrm{dd}, J=6.7,1.6 \mathrm{~Hz}, 1.47 \mathrm{H},(Z)-9 \mathrm{a}), 1.30(\mathrm{~s}, 3 \mathrm{H}), 1.26(\mathrm{~d}, J=1.7 \mathrm{~Hz}$, $3 \mathrm{H})$. The $E / Z$ ratio was determined by integration of the resonances at $\delta 4.26$ (major) and 4.18 (minor) ppm; ${ }^{13} \mathrm{C}\left\{{ }^{1} \mathrm{H}\right\} \mathrm{NMR}(101 \mathrm{MHz}$, $\left.\mathrm{CDCl}_{3}\right): \delta 146.2,145.1,139.8,139.7,128.5,121.0,120.4,101.2,98.8$, $68.2,65.7,64.0,58.4,36.2,36.2,27.1,24.9,18.8,16.6,12.7,9.3$. HRMS $\left(\mathrm{CI}^{+}\right) \mathrm{m} / z:[\mathrm{M}+\mathrm{H}]^{+}$calcd for $\mathrm{C}_{13} \mathrm{H}_{23} \mathrm{O}_{2}, 211.1693$; found, 211.1694

(S)-2,2,4,4-Tetramethyl-5-(3-methyl-6-phenylhex-3-en-1-yl)-1,3dioxolane $[(S)-8 b]$. Following the general procedure for relay crossmetathesis using acetonide $(S)-(5 d)(67 \mathrm{mg})$ and alkene $7 \mathrm{a}(200 \mathrm{mg})$ gave trisubstituted alkene $(S)-8 \mathbf{b}(51 \mathrm{mg}, 0.17 \mathrm{mmol}, 68 \%)$ as a light brown oil and a mixture of $E / Z$ geometrical isomers $(E / Z=70: 30)$. $10.14469 / \mathrm{hpc} / 6326 . R_{f} 0.58$ (5\% EtOAc in petrol); IR (ATR, neat) $3023 \mathrm{~cm}^{-1}$; ${ }^{1} \mathrm{H}$ NMR $\left(400 \mathrm{MHz} \mathrm{CDCl}_{3}\right): \delta 7.32-7.24(\mathrm{~m}, 2 \mathrm{H})$, $7.23-7.15(\mathrm{~m}, 3 \mathrm{H}), 5.29-5.20(\mathrm{~m}, 1 \mathrm{H}), 3.66(\mathrm{dd}, J=9.3,3.5 \mathrm{~Hz}, E-$ $8 \mathbf{b}, 0.70 \mathrm{H}), 3.62(\mathrm{dd}, \mathrm{J}=9.6,3.3 \mathrm{~Hz}, \mathrm{Z}-8 \mathrm{~b}, 0.30 \mathrm{H}), 2.70-2.61(\mathrm{~m}$, $2 \mathrm{H}), 2.38-2.27(\mathrm{~m}, 2 \mathrm{H}), 2.27-2.10(\mathrm{~m}, 1 \mathrm{H}), 2.09-1.96(\mathrm{~m}, 1 \mathrm{H})$, $1.74-1.68(\mathrm{~m}, Z-8 \mathrm{~b}, 0.90 \mathrm{H}), 1.67-1.59(\mathrm{~m}, 1 \mathrm{H}), 1.58(\mathrm{~s}, E-8 \mathbf{b}$, $2.10 \mathrm{H}), 1.53-1.42(\mathrm{~m}, 1 \mathrm{H}), 1.43(\mathrm{~s}, E-8 \mathrm{~b}, 2.10 \mathrm{H}), 1.43(\mathrm{~s}, \mathrm{Z}-8 \mathrm{~b}$, $0.90 \mathrm{H}), 1.33$ (s, E-8b, 2.10H), 1.32 (s, Z-8b, 0.90H), 1.24 (s, E-8b, $2.10 \mathrm{H}), 1.23$ (s, Z-8b, 0.90H), 1.11 (s, E-8b, 2.10H), 1.08 (s, Z-8b, $0.90 \mathrm{H})$. The $E / Z$ ratio was determined by integration of the resonances at $\delta 1.11$ (major) and 1.08 (minor) ppm; ${ }^{13} \mathrm{C}\left\{{ }^{1} \mathrm{H}\right\}$ NMR (101 MHz, $\left.\mathrm{CDCl}_{3}\right): \delta 142.3,135.1,128.5,128.2,125.7,125.2$, 124.1, 106.5, 82.8, 82.7, 80.1, 80.1, 36.7, 36.3, 36.1, 29.9, 29.8, 28.9, $28.6,28.6,27.8,27.6,26.9,26.1,23.3,22.9,16.0$. The E-isomer was identified as the major isomer on the basis of a characteristic shielded methyl resonance ${ }^{23}$ at 16.0 versus $23.3 \mathrm{ppm}$ for the minor $Z$-isomer; HRMS $\left(\mathrm{EI}^{+}\right) \mathrm{m} / z$ : $\left[\mathrm{M}-\mathrm{CH}_{3}\right]^{+}$calcd for $\mathrm{C}_{19} \mathrm{H}_{27} \mathrm{O}_{2}, 287.2011$; found, 287.2022. Data for $(R)-8 \mathbf{b}$ was identical.

3-(6-(4-Methoxyphenyl)-3-methylhex-3-en-1-yl)-2,2-dimethyloxirane $(8 c){ }^{2 a}$ Following the general procedure for relay crossmetathesis using epoxy allyl ether $(E)$ - or $(Z)-5 a(53 \mathrm{mg})$ and alkene $7 \mathrm{c}(250 \mathrm{mg}$, containing $5 \%$ p-methoxytoluene) gave trisubstituted alkene $8 \mathrm{c}(45 \mathrm{mg}, 0.17 \mathrm{mmol}, 66 \%$ from $(E)$-5a; 41 $\mathrm{mg}, 0.16 \mathrm{mmol}, 60 \%$ from $(Z)-5 a)$ as a brown oil and a mixture of $E /$ $Z$ geometrical isomers $[E / Z=66: 34$ (from $(E)-5 a$ ) or 67:33 (from (Z)-5a) ]. 10.14469/hpc/5817. $R_{f} 0.35$ (5\% EtOAc in petrol); ${ }^{1} \mathrm{H}$ NMR $\left(400 \mathrm{MHz}, \mathrm{CDCl}_{3}\right): \delta 7.10(\mathrm{~d}, J=8.6 \mathrm{~Hz}, 2 \mathrm{H}), 6.82(\mathrm{~d}, J=8.6$, $2 \mathrm{H}), 5.25(\mathrm{br} \mathrm{t}, J=7.2 \mathrm{~Hz}, 1 \mathrm{H}), 3.79(\mathrm{~s}, 3 \mathrm{H}), 2.72-2.66(\mathrm{~m}, 1 \mathrm{H})$, $2.63-2.54(\mathrm{~m}, 2 \mathrm{H}), 2.34-2.23(\mathrm{~m}, 2 \mathrm{H}), 2.21-2.04(\mathrm{~m}, 2 \mathrm{H}), 1.70(\mathrm{~d}$, $J=1.3 \mathrm{~Hz}, \mathrm{Z}-8 \mathrm{c}, 1 \mathrm{H}), 1.60(\mathrm{~s}, E-8 \mathrm{c}, 2 \mathrm{H}), 1.66-1.46(\mathrm{~m}, 2 \mathrm{H}), 1.30(\mathrm{~s}$, $3 \mathrm{H}), 1.26(\mathrm{~s}, E-8 \mathrm{c}, 2 \mathrm{H}), 1.25(\mathrm{~s}, Z-8 \mathrm{c}, 1 \mathrm{H})$; The $E / Z$ ratio was determined by integration of the resonances at $\delta 1.60$ (major) and 1.70 (minor) ppm; ${ }^{13} \mathrm{C}\left\{{ }^{1} \mathrm{H}\right\}$ NMR $\left(101 \mathrm{MHz}, \mathrm{CDCl}_{3}\right): \delta 157.8$, $134.9,134.9,134.5,129.4,125.3,124.4,113.8,113.8,64.3,64.2,58.4$, $55.4,36.4,35.5,35.2,30.3,30.2,28.6,27.6,27.5,25.0,25.0,23.5$, 
$18.9,18.8,16.1$. The $E$-isomer was identified as the major isomer on the basis of a characteristic shielded methyl resonance ${ }^{23}$ at 16.1 versus $23.5 \mathrm{ppm}$ for the minor $Z$-isomer; HRMS $\left(\mathrm{CI}^{+}\right) \mathrm{m} / z$ : $[\mathrm{M}+\mathrm{H}]^{+}$calcd for $\mathrm{C}_{18} \mathrm{H}_{27} \mathrm{O}_{2}, 275.2011$; found, 275.2008. Data for $(R)-8 \mathrm{c}$ was identical.

3-(6-(3-Methoxyphenyl)-3-methylhex-3-en-1-yl)-2,2-dimethyloxirane $(8 d) .^{3 d}$ Following the general procedure for relay crossmetathesis using epoxy allyl ether $(E)-5 a(9.5 \mathrm{mg}, 0.04 \mathrm{mmol}, 1.0$ equiv) alkene $7 \mathrm{~d}(44 \mathrm{mg}, 0.23 \mathrm{mmol}, 5.0$ equiv), and ruthenium benzylidene $1(3.4 \mathrm{mg}, 0.004 \mathrm{mmol}, 10 \mathrm{~mol} \%)$ gave trisubstituted alkene $8 \mathrm{~d}(7.5 \mathrm{mg}, 0.024 \mathrm{mmol}, 61 \%)$ as a colorless oil and a mixture of $E / Z$ geometrical isomers $(E / Z=72: 28) .10 .14469 / \mathrm{hpc} / 6322 . R_{f}$ 0.45 (10\% EtOAc in pentanes); ${ }^{1} \mathrm{H}$ NMR $\left(400 \mathrm{MHz}, \mathrm{CDCl}_{3}\right): \delta$ $7.23-7.14(\mathrm{~m}, 1 \mathrm{H}), 6.80-6.76(\mathrm{~m}, 1 \mathrm{H}), 6.74(\mathrm{~d}, J=1.4 \mathrm{~Hz}, 1 \mathrm{H})$, 6.75-6.70 (m, 1H), 5.31-5.12 (m, 1H), $3.80(\mathrm{~s}, 3 \mathrm{H}), 2.72-2.66(\mathrm{~m}$, $1 \mathrm{H}), 2.65-2.59(\mathrm{~m}, 2 \mathrm{H}), 2.37-2.27(\mathrm{~m}, 2 \mathrm{H}), 2.22-2.03(\mathrm{~m}, 2 \mathrm{H})$, $1.70(\mathrm{~d}, J=1.3 \mathrm{~Hz}, 0.84 \mathrm{H}, Z-8 \mathrm{~d}), 1.66-1.54(\mathrm{~m}, 2 \mathrm{H}), 1.58(\mathrm{~d}, J=1.2$ $\mathrm{Hz}, 2.16 \mathrm{H}, E-8 \mathrm{~d}), 1.30$ (s, 3H), 1.26 (s, $2.16 \mathrm{H}, E-8 \mathrm{~d}), 1.25$ (s, $0.84 \mathrm{H}$, $Z$-8d). The $E / Z$ ratio was determined by integration of the resonances at $\delta 1.58$ (major) and 1.70 (minor) ppm. ${ }^{13} \mathrm{C}\left\{{ }^{1} \mathrm{H}\right\}$ NMR (101 MHz, $\left.\mathrm{CDCl}_{3}\right): \delta 159.7,144.1,135.0,129.3,125.2,124.3,121.0,114.4$, 111.1, 64.3, 64.2, 58.5, 55.3, 36.5, 36.2, 29.9, 28.7, 27.6, 25.0, 23.5, $18.9,16.1$. The E-isomer was identified as the major isomer on the basis of a characteristic shielded methyl resonance ${ }^{23}$ at 16.1 versus $23.5 \mathrm{ppm}$ for the minor Z-isomer; HRMS $\left(\mathrm{ES}^{+}\right) \mathrm{m} / z$ : $[\mathrm{M}+\mathrm{H}]^{+}$calcd for $\mathrm{C}_{18} \mathrm{H}_{27} \mathrm{O}_{2}, 275.2011$; found, 275.2008 .

3-(6-(2-Methoxyphenyl)-3-methylhex-3-en-1-yl)-2,2-dimethyloxirane $(8 e)$. Following the general procedure for relay cross-metathesis using epoxy allyl ether $(E)-5 a(53 \mathrm{mg})$ and alkene 7 e $(238 \mathrm{mg})$ gave trisubstituted alkene $8 \mathbf{e}(54 \mathrm{mg}, 0.20 \mathrm{mmol}, 78 \%)$ as a light brown oil and a mixture of $E / Z$ geometrical isomers $(E / Z=68: 32) .10 .14469 /$ hpc/6323. $R_{f} 0.15$ (4\% EtOAc in petrol); ${ }^{1} \mathrm{H}$ NMR $(400 \mathrm{MHz}$ $\left.\mathrm{CDCl}_{3}\right): \delta 7.20-6.81(\mathrm{~m}, 4 \mathrm{H}), 5.30-5.22(\mathrm{~m}, 1 \mathrm{H}), 3.83(\mathrm{~s}, E-8 \mathrm{e}$, $2.10 \mathrm{H}), 3.82(\mathrm{~s}, \mathrm{Z}-8 \mathrm{e}, 0.90 \mathrm{H}), 2.74-2.66(\mathrm{~m}, 1 \mathrm{H}), 2.67-2.59(\mathrm{~m}$, $2 \mathrm{H}), 2.33-2.23(\mathrm{~m}, 2 \mathrm{H}), 2.20-2.02(\mathrm{~m}, 2 \mathrm{H}), 1.70(\mathrm{~d}, J=1.3 \mathrm{~Hz}, \mathrm{Z}-$ 8e, $0.90 \mathrm{H}), 1.69-1.44(\mathrm{~m}, 2 \mathrm{H}), 1.58(\mathrm{~d}, J=1.3 \mathrm{~Hz}, E-8 \mathrm{e}, 2.10 \mathrm{H})$, $1.30(\mathrm{~s}, 3 \mathrm{H}), 1.26(\mathrm{~s}, E-8 \mathrm{e}, 2.10 \mathrm{H}), 1.26(\mathrm{~s}, Z-8 \mathrm{e}, 0.90 \mathrm{H})$. The $E / Z$ ratio was determined by integration of the resonances at $\delta 1.26$ (major) and 1.26 (minor) ppm; ${ }^{13} \mathrm{C}\left\{{ }^{1} \mathrm{H}\right\}$ NMR $\left(101 \mathrm{MHz}, \mathrm{CDCl}_{3}\right.$ ): $\delta 157.5,134.5,130.6,129.8,127.0,127.0,125.7,124.8,120.3,120.3$, $110.2,64.2,64.2,58.4,55.2,36.3,30.8,30.5,28.5,28.2,27.5,24.9$, $23.4,18.8,18.7,15.9$. The $E$-isomer was identified as the major isomer on the basis of a characteristic shielded methyl resonance ${ }^{23}$ at 15.9 versus $23.4 \mathrm{ppm}$ for the minor $Z$-isomer; HRMS $\left(\mathrm{ES}^{+}\right) \mathrm{m} / \mathrm{z}$ : $[\mathrm{M}+$ $\mathrm{H}]^{+}$calcd for $\mathrm{C}_{18} \mathrm{H}_{27} \mathrm{O}_{2}$, 275.2011; found, 275.2007.

2,2-Dimethyl-3-(3-methyl-6-(p-tolyl)hex-3-en-1-yl)oxirane (8f). ${ }^{2 a}$ Following the general procedure for relay cross-metathesis using epoxy allyl ether $(E)-5 \mathbf{a}(53 \mathrm{mg})$ and alkene $7 \mathrm{f}(218 \mathrm{mg})$ gave trisubstituted alkene $8 \mathrm{f}(43 \mathrm{mg}, 0.16 \mathrm{mmol}, 65 \%)$ as a colorless oil and a mixture of $E / Z$ geometrical isomers $(E / Z=72: 28) .10 .14469 /$ $\mathrm{hpc} / 6414 . R_{f} 0.31$ (10\% EtOAc in pentanes); ${ }^{1} \mathrm{H}$ NMR $(400 \mathrm{MHz}$, $\left.\mathrm{CDCl}_{3}\right): \delta 7.09(\mathrm{~s}, 4 \mathrm{H}), 5.29-5.21(\mathrm{~m}, 1 \mathrm{H}), 2.74-2.67(\mathrm{~m}, 1 \mathrm{H})$, $2.65-2.58(\mathrm{~m}, 2 \mathrm{H}), 2.36-2.27(\mathrm{~m}, 2 \mathrm{H}), 2.33(\mathrm{~s}, 3 \mathrm{H}), 2.23-2.04(\mathrm{~m}$, $2 \mathrm{H}), 1.72(\mathrm{~d}, J=1.4 \mathrm{~Hz}, 0.84 \mathrm{H}, Z-8 \mathrm{f}), 1.72-1.51(\mathrm{~m}, 2 \mathrm{H}), 1.62(\mathrm{~s}$, $2.16 \mathrm{H}, E-8 \mathrm{f}), 1.34$ (s, 3H), 1.30 (s, 2.16H, E-8f), 1.29 (s, 0.84H, Z$8 f)$. The $E / Z$ ratio was determined by integration of the resonances at $\delta 1.62$ (major) and 1.72 (minor) $\mathrm{ppm} ;{ }^{13} \mathrm{C}\left\{{ }^{1} \mathrm{H}\right\} \mathrm{NMR}(101 \mathrm{MHz}$, $\left.\mathrm{CDCl}_{3}\right): \delta 139.3,139.2,135.2,135.2,134.8,129.1,129.0,128.4$, 125.3, 124.5, 64.2, 64.2, 58.4, 36.4, 35.9, 35.7, 30.2, 30.1, 28.6, 27.5, $27.5,25.0,25.0,23.4,21.1,18.9,18.8,16.1$. The E-isomer was identified as the major isomer on the basis of a characteristic shielded methyl resonance ${ }^{23}$ at 16.1 versus $23.4 \mathrm{ppm}$ for the minor $Z$-isomer; HRMS $\left(\mathrm{ES}^{+}\right) \mathrm{m} / z:[\mathrm{M}+\mathrm{H}]^{+}$calcd for $\mathrm{C}_{18} \mathrm{H}_{27} \mathrm{O}, 259.2062$; found, 259.2056.

3-(6-(4-Chlorophenyl)-3-methylhex-3-en-1-yl)-2,2-dimethyloxirane $(8 \mathrm{~g})$. Following the general procedure for relay cross-metathesis using epoxy allyl ether $(E)-5 a(53 \mathrm{mg})$ and alkene $7 \mathrm{~g}(244 \mathrm{mg})$ gave trisubstituted alkene $8 \mathrm{~g}(47 \mathrm{mg}, 0.17 \mathrm{mmol}, 68 \%)$ as a light brown oil and a mixture of $E / Z$ geometrical isomers $(E / Z=78: 22) .10 .14469 /$ hpc/6325. $R_{f} 0.25$ (4\% EtOAc in petrol); ${ }^{1} \mathrm{H}$ NMR (400 MHz
$\left.\mathrm{CDCl}_{3}\right): \delta 7.25-7.20(\mathrm{~m}, 2 \mathrm{H}), 7.12-7.08(\mathrm{~m}, 2 \mathrm{H}), 5.22-5.15(\mathrm{~m}$, $1 \mathrm{H}), 2.71-2.64(\mathrm{~m}, 1 \mathrm{H}), 2.64-2.56(\mathrm{~m}, 2 \mathrm{H}), 2.34-2.23(\mathrm{~m}, 2 \mathrm{H})$, $2.21-2.01(\mathrm{~m}, 2 \mathrm{H}), 1.69(\mathrm{q}, J=1.3 \mathrm{~Hz}, Z-8 \mathrm{~g}, 0.66 \mathrm{H}), 1.64-1.57(\mathrm{~m}$, $2 \mathrm{H}), 1.55$ (s, E-8g, 2.34H), 1.29 (s, 3H), 1.26 (s, E-8g, 2.34H), 1.25 $(\mathrm{s}, Z-8 \mathrm{~g}, 0.66 \mathrm{H})$. The $E / Z$ ratio was determined by integration of the resonances at $\delta 1.26$ (major) and 1.25 (minor) ppm; ${ }^{13} \mathrm{C}\left\{{ }^{1} \mathrm{H}\right\}$ NMR $\left(101 \mathrm{MHz}, \mathrm{CDCl}_{3}\right): \delta 140.6,135.3,131.4,129.8,128.3,128.3,124.6$, $123.8,64.1,58.3,36.3,35.6,35.3,29.7,29.7,28.5,27.4,27.3,24.9$, $23.3,18.7,16.0$. The $E$-isomer was identified as the major isomer on the basis of a characteristic shielded methyl resonance ${ }^{23}$ at 16.0 versus $23.3 \mathrm{ppm}$ for the minor $Z$-isomer; HRMS $\left(\mathrm{CI}^{+}\right) \mathrm{m} / z$ : $[\mathrm{M}+\mathrm{H}]^{+}$calcd for $\mathrm{C}_{17} \mathrm{H}_{24} \mathrm{O}^{35} \mathrm{Cl}$, 279.1510; found, 279.1510.

2,2-Dimethyl-3-(3-methyldec-3-en-1-yl)oxirane (8h). Following the general procedure for relay cross-metathesis using epoxy allyl ether $(E)-5 a(53 \mathrm{mg})$ and alkene $7 \mathrm{~h}(175 \mathrm{mg})$ gave trisubstituted alkene $8 \mathrm{~h}(51 \mathrm{mg}, 0.23 \mathrm{mmol}, 92 \%)$ as a colorless oil and a mixture of $E / Z$ geometrical isomers $(E / Z=70: 30) .10 .14469 / \mathrm{hpc} / 5762 . R_{f} 0.57$ (10\% $\mathrm{Et}_{2} \mathrm{O}$ in pentanes); ${ }^{1} \mathrm{H}$ NMR (400 MHz, Acetone-d6): $\delta 5.24-$ $5.14(\mathrm{~m}, 1 \mathrm{H}), 2.64(\mathrm{t}, J=6.3 \mathrm{~Hz}, Z-8 \mathrm{~h}, 0.3 \mathrm{H}), 2.61(\mathrm{t}, J=6.2 \mathrm{~Hz}, E-$ $8 \mathrm{~h}, 0.7 \mathrm{H}), 2.24-1.95(\mathrm{~m}, 4 \mathrm{H}), 1.72-1.52(\mathrm{~m}, 5 \mathrm{H}), 1.38-1.25(\mathrm{~m}$, $8 \mathrm{H}), 1.23(\mathrm{~s}, Z-8 \mathrm{~h}, 0.9 \mathrm{H}), 1.23-1.22(\mathrm{~m}, 3 \mathrm{H}), 1.21(\mathrm{~s}, E-8 \mathrm{~h}, 2.1 \mathrm{H})$, $0.92-0.85(\mathrm{~m}, 3 \mathrm{H})$. The $E / Z$ ratio was determined by integration of the resonances at $\delta 1.21$ (major) and 1.23 (minor) ppm; ${ }^{13} \mathrm{C}\left\{{ }^{1} \mathrm{H}\right\}$ NMR $\left(101 \mathrm{MHz}\right.$, DMSO- $\left.d_{6}\right): \delta 133.8,125.6,124.7,62.8,62.7,57.6$, 57.5, 35.9, 31.2, 29.5, 29.3, 28.4, 28.3, 28.0, 27.3, 27.2, 26.9, 24.6 $24.6,23.1,22.1,18.5,18.5,15.7,13.9$. The $E$-isomer was identified as the major isomer on the basis of a characteristic shielded methyl resonance $^{23}$ at 15.7 versus $23.1 \mathrm{ppm}$ for the minor $Z$-isomer; HRMS $\left(\mathrm{CI}^{+}\right) \mathrm{m} / z:[\mathrm{M}+\mathrm{H}]^{+}$calcd for $\mathrm{C}_{15} \mathrm{H}_{29} \mathrm{O}$, 225.2213; found, 225.2212.

8-(3,3-Dimethyloxiran-2-yl)-6-methyloct-5-en-2-one (8i). ${ }^{42}$ Following the general procedure for relay cross-metathesis using epoxy allyl ether $(E)-5 a(53 \mathrm{mg})$ and alkene $7 \mathbf{l}(0.18 \mathrm{~mL})$ gave trisubstituted alkene $8 \mathbf{i}$ ( $34 \mathrm{mg}, 0.16 \mathrm{mmol}, 64 \%$ ) as a light brown oil and a mixture of $E / Z$ geometrical isomers $(E / Z=73: 27) .10 .14469 / \mathrm{hpc} / 5764 . R_{f}$ $0.21\left(20 \% \mathrm{Et}_{2} \mathrm{O}\right.$ in pentanes); IR (ATR, neat) $1715 \mathrm{~cm}^{-1} ;{ }^{1} \mathrm{H}$ NMR $\left(400 \mathrm{MHz}, \mathrm{CDCl}_{3}\right): \delta 5.15-5.07(\mathrm{~m}, 1 \mathrm{H}), 2.69(\mathrm{t}, J=6.3 \mathrm{~Hz}, \mathrm{Z}-8 \mathrm{i}$, $0.27 \mathrm{H}), 2.65(\mathrm{t}, J=6.3 \mathrm{~Hz}, E-8 \mathrm{i}, 0.73 \mathrm{H}), 2.47-2.41(\mathrm{~m}, 2 \mathrm{H}), 2.29-$ $2.21(\mathrm{~m}, 2 \mathrm{H}), 2.12(\mathrm{~s}, 3 \mathrm{H}), 2.19-2.00(\mathrm{~m}, 2 \mathrm{H}), 1.68-1.56(\mathrm{~m}, 5 \mathrm{H})$, $1.29(\mathrm{~s}, Z-8 \mathrm{i}, 0.81 \mathrm{H}), 1.28$ (s, E-8i, $2.19 \mathrm{H}), 1.25(\mathrm{~s}, Z-8 \mathrm{i}, 0.81 \mathrm{H}), 1.24$ $(\mathrm{s}, E-8 \mathbf{i}, 2.19 \mathrm{H})$. The $E / Z$ ratio was determined by integration of the resonances at $\delta 1.24$ (major) and 1.25 (minor) ppm; ${ }^{13} \mathrm{C}\left\{{ }^{1} \mathrm{H}\right\} \mathrm{NMR}$ $\left(101 \mathrm{MHz}, \mathrm{CDCl}_{3}\right): \delta 208.8,135.6,135.6,124.2,123.3,64.2,64.1$, $58.5,58.4,43.9,43.8,36.4,30.1,30.1,28.6,27.5,27.5,25.0,25.0$, $23.4,22.5,22.3,18.9,18.9,16.1$. The $E$-isomer was identified as the major isomer on the basis of a characteristic shielded methyl resonance $^{23}$ at 16.1 versus $23.4 \mathrm{ppm}$ for the minor $Z$-isomer; HRMS $\left(\mathrm{ES}^{+}\right) \mathrm{m} / z:[\mathrm{M}+\mathrm{H}]^{+}$calcd for $\mathrm{C}_{13} \mathrm{H}_{23} \mathrm{O}_{2}, 211.1693$; found, 211.1693 .

(S)-2,6-Dimethylhept-6-ene-2,3-diol (5i). ${ }^{43}$ Following the general procedure for relay cross-metathesis using diol $(S)-5 \mathbf{b}(57 \mathrm{mg})$ and alkene $7 \mathbf{a}(200 \mathrm{mg})$ gave truncated alkene $5 \mathbf{i}(10 \mathrm{mg}, 0.06 \mathrm{mmol}$, $24 \%)$ as an inseparable $85: 15$ mixture containing $(S, 6 E)$-2,6-dimethyl8-(prop-1-en-1-yloxy)oct-6-ene-2,3-diol (10b) $(E / Z=2: 1)$. Vinyl ether 10b was identified by comparison of spectroscopic data with epoxide analogue 10a, and the $E / Z$ ratio was determined by ${ }^{1} \mathrm{H}$ NMR and assigned on the basis of characteristic ${ }^{3} \mathrm{~J}_{\mathrm{H}-\mathrm{H}}$ coupling constants. $10.14469 / \mathrm{hpc} / 5765 . R_{f} 0.40$ (40\% EtOAc in petrol); IR (ATR, neat) $3600-3100,3075,1648 \mathrm{~cm}^{-1}$; ${ }^{1} \mathrm{H}$ NMR $\left(400 \mathrm{MHz}, \mathrm{CDCl}_{3}\right): \delta 6.22$ $(\mathrm{dq}, J=12.6,1.6 \mathrm{~Hz}, 0.1 \mathrm{H}, E-10 \mathrm{~b}), 5.96(\mathrm{dq}, J=6.2,1.6 \mathrm{~Hz}, 0.05 \mathrm{H}$, $Z-10 b), 5.42(\mathrm{tq}, J=6.7,1.3 \mathrm{~Hz}, 0.15 \mathrm{H}, 10 \mathrm{~b}), 4.84-4.76(\mathrm{~m}, 0.1 \mathrm{H}$, 10b), $4.74(\mathrm{~s}, 0.85 \mathrm{H}), 4.73(\mathrm{~s} 0.85 \mathrm{H}), 4.44-4.35(\mathrm{~m}, 0.05 \mathrm{H}, \mathrm{Z}-10 \mathrm{~b})$, $4.26(\mathrm{~d}, J=6.7 \mathrm{~Hz}, 0.1 \mathrm{H}, Z-10 \mathrm{~b}), 4.18(\mathrm{~d}, J=6.7 \mathrm{~Hz}, 0.2 \mathrm{H}, E-10 \mathrm{~b})$, $3.36(\mathrm{~d}, J=10.6 \mathrm{~Hz}, 0.85 \mathrm{H}), 3.34(\mathrm{~d}, J=11.9 \mathrm{~Hz}, 0.15 \mathrm{H}, 10 \mathrm{~b}), 2.38-$ $2.20(\mathrm{~m}, 2 \mathrm{H}), 2.16-2.01(\mathrm{~m}, 2 \mathrm{H}), 1.74(\mathrm{~d}, J=1.2 \mathrm{~Hz}, 2.6 \mathrm{H}), 1.69(\mathrm{~d}$, $J=1.4 \mathrm{~Hz}, 0.5 \mathrm{H}, 10 \mathrm{~b}), 1.67-1.53(\mathrm{~m}, 1.5 \mathrm{H}), 1.50-1.39(\mathrm{~m}, 1 \mathrm{H})$, $1.21(\mathrm{~s}, 2.5 \mathrm{H}), 1.20(\mathrm{~s}, 0.5 \mathrm{H}, 10 \mathrm{~b}), 1.16(\mathrm{~s}, 2.5 \mathrm{H}), 1.15(\mathrm{~s}, 0.5 \mathrm{H}$, 10b). For $5 \mathbf{i}$ only: ${ }^{13} \mathrm{C}\left\{{ }^{1} \mathrm{H}\right\}$ NMR $\left(101 \mathrm{MHz}, \mathrm{CDCl}_{3}\right): \delta 145.9,110.5$, 79.4, 72.7, 35.0, 29.6, 26.6, 23.4, 22.6; HRMS $\left(\mathrm{ES}^{+}\right) \mathrm{m} / z:[\mathrm{M}-\mathrm{OH}]^{+}$ calcd for $\mathrm{C}_{9} \mathrm{H}_{17} \mathrm{O}, 141.1279$; found, 141.1283.

(S)- and (R)-4,4-Dimethyl-5-(3-methyl-6-phenylhex-3-en-1-yl)-2phenyl-1,3,2-dioxaborolane [(S)-8j and (R)-8j]. Following the 
general procedure for relay cross-metathesis using boronates $(S)$ - or (R)-5g (59 mg, $0.19 \mathrm{mmol}, 1.0$ equiv), alkene $7 \mathrm{a}(150 \mathrm{mg}, 0.94$ mmol, 5.0 equiv), and ruthenium benzylidene $1(16 \mathrm{mg}, 0.019 \mathrm{mmol}$, $10 \mathrm{~mol} \%)$ gave trisubstituted alkenes $(S)-8 \mathbf{j}(38 \mathrm{mg}, 0.11 \mathrm{mmol}$, $58 \%)$ or $(R)-8 \mathbf{j}(44 \mathrm{mg}, 0.13 \mathrm{mmol}, 68 \%)$ as brown oils and a mixture of $E / Z$ geometrical isomers $[(S)-8 \mathbf{j}: E / Z=60: 40 ;(R)-8 \mathbf{j}: E / Z=$ 62:38]. $10.14469 / \mathrm{hpc} / 6328 . R_{f} 0.40$ (5\% EtOAc in petroleum ether, streaking); ${ }^{1} \mathrm{H}$ NMR $\left(400 \mathrm{MHz}, \mathrm{CDCl}_{3}\right): \delta 7.85-7.78(\mathrm{~m}, 2 \mathrm{H})$, $7.50-7.32(\mathrm{~m}, 3 \mathrm{H}), 7.30-7.14(\mathrm{~m}, 5 \mathrm{H}), 5.32-5.23(\mathrm{~m}, 1 \mathrm{H}), 4.03$ (dd, $J=10.0,3.5 \mathrm{~Hz}, E-8 \mathbf{j}, 0.60 \mathrm{H}), 3.98$ (dd, $J=10.4,3.2 \mathrm{~Hz}, Z-8 \mathbf{j}$, $0.40 \mathrm{H}), 2.69-2.62(\mathrm{~m}, 2 \mathrm{H}), 2.44-2.06(\mathrm{~m}, 4 \mathrm{H}), 1.72(\mathrm{~d}, J=1.3 \mathrm{~Hz}$, $Z-8 \mathbf{j}, 1.20 \mathrm{H}), 1.74-1.63(\mathrm{~m}, 1 \mathrm{H}), 1.59(\mathrm{~s}, E-8 \mathbf{j}, 1.80 \mathrm{H}), 1.62-1.47$ (m, 1H), 1.42 (s, E-8j, 1.80H), 1.41 (s, Z-8j, 1.20H), 1.28 (s, E-8j, $1.80 \mathrm{H}), 1.25(\mathrm{~s}, Z-8 \mathbf{j}, 1.20 \mathrm{H})$. The $E / Z$ ratio was determined by integration of the resonances at $\delta 1.28$ (major) and 1.25 (minor) ppm; ${ }^{13} \mathrm{C}\left\{{ }^{1} \mathrm{H}\right\}$ NMR $\left(101 \mathrm{MHz}, \mathrm{CDCl}_{3}\right): \delta 142.3,135.1,135.0$, $134.8,131.3,128.5,128.5,128.2,127.7,125.7,125.4,124.2,85.2$, $85.1,82.1,82.0,36.4,36.1,30.1,30.0,29.8,28.8,28.7,28.6,23.5$, $23.4,23.3,16.1$. The $E$-isomer was identified as the major isomer on the basis of a characteristic shielded methyl resonance ${ }^{23}$ at 16.1 versus $23.3 \mathrm{ppm}$ for the minor $Z$-isomer; HRMS $\left(\mathrm{CI}^{+}\right) \mathrm{m} / z$ : $[\mathrm{M}+\mathrm{H}]^{+}$calcd for $\mathrm{C}_{23} \mathrm{H}_{30}{ }^{11} \mathrm{BO}_{2}, 349.2333$; found, 349.2332 .

(S)- and (R)-6-Methyl-8-(2,2,5,5-tetramethyl-1,3-dioxolan-4-yl)oct-5-en-2-one $[(S)-8 \boldsymbol{k} \text { and }(R)-8 k]^{39}{ }^{3}$ Following the general procedure for relay cross-metathesis using acetonide $(S)$ - or $(R)-\mathbf{5 d}$ $(67 \mathrm{mg})$ and alkene $71(158 \mathrm{mg})$ gave trisubstituted alkenes $(S)-8 \mathbf{k}$ (46 mg, $0.17 \mathrm{mmol}, 69 \%$ ) or (R)-8k (42 mg, $0.16 \mathrm{mmol}, 62 \%$ ) as colorless oils and a mixture of $E / Z$ geometrical isomers $[(S)-8 \mathbf{k}: E / Z$ $=73: 27 ;(R)-8 \mathrm{k}: E / Z=70: 30] .10 .14469 / \mathrm{hpc} / 5767 . R_{f} 0.24(10 \%$ EtOAc in petrol); IR (ATR, neat) $1715 \mathrm{~cm}^{-1} ;{ }^{1} \mathrm{H}$ NMR (400 MHz, $\left.\mathrm{CDCl}_{3}\right): \delta 5.14-5.07(\mathrm{~m}, 1 \mathrm{H}), 3.61(\mathrm{dd}, J=9.4,3.4 \mathrm{~Hz}, 1 \mathrm{H}), 2.48-$ $2.38(\mathrm{~m}, 2 \mathrm{H}), 2.30-2.10(\mathrm{~m}, 3 \mathrm{H}), 2.11(\mathrm{~s}, 3 \mathrm{H}), 2.04-1.93(\mathrm{~m}, 1 \mathrm{H})$, $1.69-1.64(\mathrm{~m}, Z-8 \mathrm{k}, 0.81 \mathrm{H}), 1.61(\mathrm{~s}, E-8 \mathrm{k}, 2.19 \mathrm{H}), 1.60-1.51(\mathrm{~m}$, $1 \mathrm{H}), 1.48-1.40(\mathrm{~m}, 1 \mathrm{H}), 1.39(\mathrm{~d}, J=0.8 \mathrm{~Hz}, 3 \mathrm{H}), 1.31-1.28(\mathrm{~m}$, $3 \mathrm{H}), 1.23-1.20(\mathrm{~m}, 3 \mathrm{H}), 1.06(\mathrm{~s}, 3 \mathrm{H})$. The $E / Z$ ratio was determined by integration of the resonances at $\delta 1.61$ (major) and 1.69-1.64 (minor) ppm; ${ }^{13} \mathrm{C}\left\{{ }^{1} \mathrm{H}\right\}$ NMR $\left(101 \mathrm{MHz}, \mathrm{CDCl}_{3}\right): \delta$ 208.6, 135.7, 124.1, 123.0, 121.3, 106.5, 106.4, 82.8, 82.6, 80.1, 43.9, 43.7, 36.6, $29.9,28.9,28.6,28.6,27.6,27.5,26.8,26.0,26.0,23.2,22.9,22.4$, $22.2,16.0$. The $E$-isomer was identified as the major isomer on the basis of a characteristic shielded methyl resonance ${ }^{23}$ at 16.0 versus $23.2 \mathrm{ppm}$ for the minor Z-isomer; HRMS $\left(\mathrm{EI}^{+}\right) \mathrm{m} / z$ : $[\mathrm{M}]^{\bullet+}$ calcd for $\mathrm{C}_{16} \mathrm{H}_{28} \mathrm{O}_{3}, 268.2038$; found, 268.2045 .

(S)- and (R)-8-(5,5-Dimethyl-2-phenyl-1,3,2-dioxaborolan-4-yl)6-methyloct-5-en-2-one $[(S)-8 I$ and $(R)-8 I]$. Following the general procedure for relay cross-metathesis using boronates $(S)$ - or $(R)-\mathbf{5 g}$ (59 mg, $0.19 \mathrm{mmol}, 1.0$ equiv), alkene 71 (118 mg, $0.94 \mathrm{mmol}, 5.0$ equiv), and ruthenium benzylidene $1(16 \mathrm{mg}, 10 \mathrm{~mol} \%)$ gave trisubstituted alkenes $(S)-81(32 \mathrm{mg}, 0.10 \mathrm{mmol}, 55 \%)$ or $(R)-81$ (35 $\mathrm{mg}, 0.11 \mathrm{mmol}, 60 \%)$ as brown oils and a mixture of $E / Z$ geometrical isomers $((S)-81: E / Z=74: 26$; $(R)-81: E / Z=76: 24) .10 .14469 / \mathrm{hpc} /$ 6329. $R_{f} 0.10$ (10\% EtOAc in petrol); IR (ATR, neat) $1715 \mathrm{~cm}^{-1} ;{ }^{1} \mathrm{H}$ NMR (400 MHz, $\left.\mathrm{CDCl}_{3}\right): \delta 7.84-7.79(\mathrm{~m}, 2 \mathrm{H}), 7.49-7.43(\mathrm{~m}, 1 \mathrm{H})$, 7.40-7.34 (m, 2H), $5.17(\mathrm{~m}, 1 \mathrm{H}), 4.04(\mathrm{dd}, J=10.0,3.4 \mathrm{~Hz}, E-8 \mathrm{l}$, $0.76 \mathrm{H}), 4.03(\mathrm{dd}, J=10.0,3.4 \mathrm{~Hz}, Z-8 \mathrm{l}, 0.24 \mathrm{H}), 2.48(\mathrm{t}, J=7.4 \mathrm{~Hz}$, $2 \mathrm{H}), 2.38-2.26(\mathrm{~m}, 3 \mathrm{H}), 2.16-2.05(\mathrm{~m}, 1 \mathrm{H}), 2.14(\mathrm{~s}, E-8 \mathrm{l}, 2.28 \mathrm{H})$, $2.11(\mathrm{~s}, Z-81,0.72 \mathrm{H}), 1.80-1.52(\mathrm{~m}, 2 \mathrm{H}), 1.71(\mathrm{~d}, J=1.3 \mathrm{~Hz}, Z-81$, $0.72 \mathrm{H}), 1.67$ (d, $J=1.3 \mathrm{~Hz}, E-8 \mathrm{l}, 2.28 \mathrm{H}), 1.44(\mathrm{~s}, Z-8 \mathrm{l}, 0.72 \mathrm{H}), 1.43$ (s, E-81, $2.28 \mathrm{H}), 1.28(\mathrm{~s}, 3 \mathrm{H})$. The $E / Z$ ratio was determined by integration of the resonances at $\delta 1.67$ (major) and 1.71 (minor) ppm; ${ }^{13} \mathrm{C}\left\{{ }^{1} \mathrm{H}\right\}$ NMR $\left(101 \mathrm{MHz}, \mathrm{CDCl}_{3}\right): \delta 208.8,208.7,135.7$, 135.6, 134.8, 131.3, 131.3, 127.8, 127.7, 124.3, 123.1, 85.3, 85.1, 82.1, 82.1, 43.9, 43.7, 36.4, 30.1, 30.0, 29.9, 29.8, 28.8, 28.5, 23.4, 23.4, $23.3,22.4,22.3,16.1$. The $E$-isomer was identified as the major isomer on the basis of a characteristic shielded methyl resonance ${ }^{23}$ at 16.1 versus $23.3 \mathrm{ppm}$ for the minor $Z$-isomer; $\mathrm{HRMS}\left(\mathrm{CI}^{+}\right) \mathrm{m} / z$ : $[\mathrm{M}+$ $\mathrm{H}]^{+}$calcd for $\mathrm{C}_{19} \mathrm{H}_{28}{ }^{11} \mathrm{BO}_{3}, 315.2126$; found, 315.2117.

(2R,4aR,10aS)-6-Methoxy-1,1,4a-trimethyl-1,2,3,4,4a,9,10,10aoctahydrophenanthren-2-ol (11). ${ }^{2 a}$ To a solution of epoxy alkene (R)-8c (50 mg, $0.18 \mathrm{mmol}, 1.0$ equiv) in $\mathrm{CH}_{2} \mathrm{Cl}_{2}(1.8 \mathrm{~mL})$ at $-78{ }^{\circ} \mathrm{C}$ was added dropwise boron trifluoride diethyl etherate $(44 \mu \mathrm{L}, 0.36$ $\mathrm{mmol}, 2.0$ equiv) and the reaction mixture was stirred at $-78^{\circ} \mathrm{C}$ for 1 $\mathrm{h}$ before allowing to warm to room temperature. The reaction mixture was quenched by the addition of saturated aqueous $\mathrm{NaHCO}_{3}(30$ $\mathrm{mL})$, extracted with $\mathrm{CH}_{2} \mathrm{Cl}_{2}(3 \times 10 \mathrm{~mL})$, dried $\left(\mathrm{Na}_{2} \mathrm{SO}_{4}\right)$, evaporated, and chromatographed $(10-15 \%$ EtOAc in petrol) to give tricycle $11(18.2 \mathrm{mg}, 0.066 \mathrm{mmol}, 56 \%$ from $(R, E)-8 \mathrm{c})$ as a colorless viscous oil. $10.14469 / \mathrm{hpc} / 6333 . R_{f} 0.33(20 \% \mathrm{EtOAc}$ in petrol); $[\mathrm{a}]_{\mathrm{D}}^{26}+34.2\left(c 1.0, \mathrm{CHCl}_{3}\right)$; IR (ATR, neat) 3600-3200 $\mathrm{cm}^{-1} ;{ }^{1} \mathrm{H}$ NMR $\left(400 \mathrm{MHz}, \mathrm{CDCl}_{3}\right): \delta 6.97(\mathrm{~d}, J=8.4 \mathrm{~Hz}, 1 \mathrm{H}), 6.79$ $(\mathrm{d}, J=2.7 \mathrm{~Hz}, 1 \mathrm{H}), 6.67(\mathrm{dd}, J=8.4,2.7 \mathrm{~Hz}, 1 \mathrm{H}), 3.77(\mathrm{~s}, 3 \mathrm{H})$, $3.38-3.25(\mathrm{~m}, 1 \mathrm{H}), 2.91$ (ddd, $J=16.8,6.6,1.5 \mathrm{~Hz}, 1 \mathrm{H}), 2.79$ (ddd, $J$ $=16.8,11.8,7.1 \mathrm{~Hz}, 1 \mathrm{H}), 2.27(\mathrm{dt}, J=13.0,3.5 \mathrm{~Hz}, 1 \mathrm{H}), 1.93-1.68$ $(\mathrm{m}, 4 \mathrm{H}), 1.57(\mathrm{dd}, J=13.0,4.7 \mathrm{~Hz}, 1 \mathrm{H}), 1.38(\mathrm{~d}, J=5.8 \mathrm{~Hz}, 1 \mathrm{H})$, $1.32(\mathrm{dd}, J=12.2,2.4 \mathrm{~Hz}, 1 \mathrm{H}), 1.20(\mathrm{~s}, 3 \mathrm{H}), 1.07(\mathrm{~s}, 3 \mathrm{H}), 0.90(\mathrm{~s}$, $3 \mathrm{H}) ;{ }^{13} \mathrm{C}\left\{{ }^{1} \mathrm{H}\right\}$ NMR $\left(101 \mathrm{MHz}, \mathrm{CDCl}_{3}\right): \delta 157.7,150.6,129.8$, 127.3, 111.0, 110.2, 78.7, 55.3, 49.8, 39.0, 37.8, 36.9, 29.8, 28.2, 28.0, 24.8, 18.9, 15.4; HRMS $\left(\mathrm{ES}^{+}\right) \mathrm{m} / z$ : $[\mathrm{M}+\mathrm{H}]^{+}$calcd for $\mathrm{C}_{18} \mathrm{H}_{27} \mathrm{O}_{2}$, 275.2011; found, 275.2018 .

\section{ASSOCIATED CONTENT}

\section{Supporting Information}

The Supporting Information is available free of charge at https://pubs.acs.org/doi/10.1021/acs.joc.0c00067.

Copies of ${ }^{1} \mathrm{H}$ and ${ }^{13} \mathrm{C}$ NMR spectra for all compounds and HPLC chromatograms for enantiomeric excess determinations of $(S)$ - and (R)-5c and (S)- and (R)5g (PDF)

\section{AUTHOR INFORMATION}

\section{Corresponding Author}

D. Christopher Braddock - Department of Chemistry, Molecular Sciences Research Hub, Imperial College London,

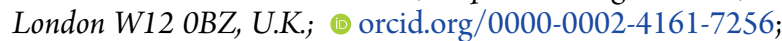
Email: c.braddock@imperial.ac.uk

\section{Authors}

Karim A. Bahou - Department of Chemistry, Molecular Sciences Research Hub, Imperial College London, London W12 OBZ, U.K.; (1) orcid.org/0000-0002-8302-5283

Adam G. Meyer - CSIRO Manufacturing, Jerry Price Laboratory, Clayton 3168, Victoria, Australia

G. Paul Savage - CSIRO Manufacturing, Jerry Price Laboratory, Clayton 3168, Victoria, Australia; 이이이.org/ 0000-0001-7805-8630

Zhensheng Shi - Department of Chemistry, Molecular Sciences Research Hub, Imperial College London, London W12 OBZ, U.K.; $\odot$ orcid.org/0000-0001-6320-4201

Tianyou He - Department of Chemistry, Molecular Sciences Research Hub, Imperial College London, London W12 OBZ, U.K.

Complete contact information is available at: https://pubs.acs.org/10.1021/acs.joc.0c00067

\section{Notes}

The authors declare no competing financial interest.

\section{ACKNOWLEDGMENTS}

We thank CSIRO and Imperial College London for a studentship (to K.A.B.), the China Scholarship Council for a scholarship (to Z.S.) and the EPSRC (grant no. EP/P030742/ 1 to D.C.B.) for financial support. 


\section{REFERENCES}

(1) Review: (a) Barrett, A.; Ma, T.-K.; Mies, T. Recent Developments in Polyene Cyclizations and Their Applications in Natural Product Synthesis. Synthesis 2019, 51, 67-82 and references cited therein.

(2) For representative examples see: (a) Zhao, J.-F.; Zhao, Y.-J.; Loh, T.-P. Indium tribromide-promoted arene-terminated epoxy olefin cyclization. Chem. Commun. 2008, 1353-1355 and references cited therein. (b) Song, L.; Zhu, G.; Liu, Y.; Liu, B.; Qin, S. Total Synthesis of Atisane-Type Diterpenoids: Application of Diels-Alder Cycloadditions of Podocarpane-Type Unmasked ortho-Benzoquinones. J. Am. Chem. Soc. 2015, 137, 13706-13714. (c) Tian, Y.; Xu, X.; Zhang, L.; $\mathrm{Qu}, \mathrm{J}$. Tetraphenylphosphonium tetrafluoroborate/1,1,1,3,3,3Hexafluoroisopropanol $\left(\mathrm{Ph}_{4} \mathrm{PBF}_{4} / \mathrm{HFIP}\right)$ Effecting Epoxide-Initiated Cation-Olefin Polycylizations. Org. Lett. 2016, 18, 268-271. (d) Mai, D.; Uchenik, D.; Vanderwal, C. Efforts Toward a Synthesis of Crotogoudin and Crotobarin. Synlett 2017, 28, 1758-1762. (e) Yang, L.-F.; Xing, Y.; Xiao, J.-X.; Xie, J.; Gao, W.; Xie, J.; Wang, L.-T.; Wang, J.; Liu, M.; Yi, Z.; Qiu, W.-W. Synthesis of Cyanoenone-Modified Diterpenoid Analogs as novel Bmi-1-Mediated Antitumor Agents. ACS Med. Chem. Lett. 2018, 9, 1105-1110.

(3) (a) Gansäuer, A.; Justicia, J.; Rosales, A.; Rinker, B. Efficient and Stereoselective Synthesis of Precursors for Epoxypolyene Cyclisations via Allylic Substitutions. Synlett 2005, 2005, 1954-1956. (b) Rosales, V.; Zambrano, J. L.; Demuth, M. Regioselective Palladium-Catalyzed Alkylation of Allylic Halides with Benzylic Grignard Reagents. TwoStep Synthesis of Abietane Terpenes and Tetracyclic Polyprenoid Compounds. J. Org. Chem. 2002, 67, 1167-1170. (c) Steglich, W.; Lang, M. An Effective method for the Synthesis of 13C-Labeled Polyprenylhydroxybenzoic Acids. Synthesis 2005, 2005, 1019-1027. (d) Cherney, E. C.; Green, J. C.; Baran, P. S. Synthesis of ent-Kaurane and Beyerane Diterpenoids by Controlled Fragmentations of Overbred Intermediates. Angew. Chem., Int. Ed. 2013, 52, 90199022. See also refs $2 a-e$.

(4) See, for example: (a) Braddock, D. C.; Marklew, J. S.; Foote, K. M.; White, A. J. P. An Enantiospecific Polyene Cyclization Initiated by an Enantiomerically Pure Bromonium Ion. Chirality 2013, 25, 692700. An alternative approach uses dithiane methodology to unite the geranyl unit with an aromatic headgroup: (b) Kim, M. B.; Shaw, J. T. Synthesis of Antimicrobial Natural products Targeting FtzA: (+)-Totarol and Related Totarane Diterpenes. Org. Lett. 2010, 12, 3324-3327. (c) Onyango, E. O.; Fu, L.; Gribble, G. W. Synthesis of a Dicyano Abietane, a Key Intermediate for the Anti-inflammatory Agent TBE-31. Org. Lett. 2014, 16, 322-324. (d) Saito, Y.; Goto, M.; Nakagawa-Goto, K. Total Synthesis of Antiproliferative Parvifloron F. Org. Lett. 2018, 20, 628-631.

(5) Warren, S. Organic Synthesis: The Disconnection Approach; John Wiley \& Sons, 1982.

(6) (a) For the original relay strategy as applied to relay ring closing metathesis see: Hoye, T. R.; Jeffrey, C. S.; Tennakoon, M. A.; Wang, J.; Zhao, H. Relay Ring-Closing Metathesis (RRCM): A Strategy for Directing Metal Movement Throughout Olefin Metathesis Sequences. J. Am. Chem. Soc. 2004, 126, 10210-10211. . See also: (b) Wallace, D. J. Relay Ring-Closing Metathesis-A Strategy for Achieving Reactivity and Selectivity in Metathesis Chemistry. Angew. Chem., Int. $E d$. 2005, 44, 1912-1915. and references therein. For the use of a modified allyl geranyl ether fragment in a relay ring closing metathesis see: (c) Lee, J.; Parker, K. A. A Formal Synthesis of (-)-Englerin A by Relay Ring Closing Metathesis and Transannular Etherification. Org. Lett. 2012, 14, 2682-2685.

(7) Ogba, O. M.; Warner, N. C.; O'Leary, D. J.; Grubbs, R. H. Recent Advances in Ruthenium-Based Olefin Metathesis. Chem. Soc. Rev. 2018, 47, 4510-4544.

(8) Ritter, T.; Hejl, A.; Wenzel, A. G.; Funk, T. W.; Grubbs, R. H. A Standard System of Characterization for Olefin Metathesis Catalysts. Organometallics 2006, 25, 5740-5745.

(9) (a) For $E$ - and Z-trisubstituted olefins by cross metathesis using molybdenum alkylidene pre-catalysts see: Nguyen, T. T.; Koh, M. J.; Mann, T. J.; Schrock, R. R.; Hoveyda, A. H. Synthesis of E- and Z- trisubstituted alkenes by catalytic cross metathesis. Nature 2017, 552 , 347-354. (b) For the formation of $E$ - and Z-trisubstituted allylic alcohols using a ruthenium catechothiolate complex see: Xu, C.; Liu, Z.; Torker, S.; Shen, X.; Xu, D.; Hoveyda, A. H. Synthesis of Z- or Etrisubstituted allylic alcohols and ethers by kinetically controlled cross-metathesis with a Ru catechothiolate complex. J. Am. Chem. Soc. 2017, 139, 15640-15643. (c) For $\alpha, \beta$-unsaturated carbonyl trisubstituted olefins by ruthenium benzylidene catalyzed crossmetathesis of citronellal, citronellol and citral with methyl methacrylate see: Bilel, H.; Hamdi, N.; Zagrouba, F.; Fischmeister, C.; Bruneau, C. Cross-metathesis transformations of terpenoids in dialkyl carbonate solvents. Green Chem. 2011, 13, 1448-1452. (d) For a trisubstituted olefin by ruthenium benzylidene catalyzed crossmetathesis of citronellal with methacrolein see: $\mathrm{Xu}$, J.; Caro-Diaz, E. J. E.; Trzoss, L.; Theodorakis, E. A. Nature-Inspired Total Synthesis Of (-)-Fusarisetin A. J. Am. Chem. Soc. 2012, 134, 5072-5075. (e) For a two-step synthesis of $(E)$-trisubstituted olefins via enyne cross metathesis followed by 1,4-hydrogenation see: Schmalz, H.-G.; Ratsch, F. An Atom-Economic and Stereospecific Access to Trisubstituted Olefins through Enyne Cross Metathesis Followed by 1,4-Hydrogenation. Synlett 2018, 29, 785-792. (f) See also ref 21.

(10) Chatterjee, A. K.; Grubbs, R. H. Synthesis of Trisubstituted Alkenes via Olefin Cross Metathesis. Org. Lett. 1999, 1, 1751-1753.

(11) (a) Grubbs subsequently showed that 2-methyl-2-butene could also be employed: Chatterjee, A. K.; Sanders, D. P.; Grubbs, R. H. Synthesis of Symmetrical Trisubstituted Olefins by Cross Metathesis. Org. Lett. 2002, 4, 1939-1942. For subsequent representative utilizations of this methodology see: (b) Magolan, J.; Coster, M. J. Total Synthesis of (+)-Angelmarin. J. Org. Chem. 2009, 74, 50835086. (c) Hodgson, D. M.; Angrish, D.; Erickson, S. P.; Kloesges, J.; Lee, C. H. Consecutive Alkene Cross-Metathesis/Oxonium Ylide Formation-Rearrangement: Synthesis of the Anti-HIV Agent Hyperolactone C. Org. Lett. 2008, 10, 5553-5556. (d) Zhang, Y.; Gaekwad, J.; Wolfert, M. A.; Boons, G.-J. Synthetic Tetra-Acylated Derivatives of Lipid A from Porphyromonas Gingivalis are Antagonists of Human TLR4. Org. Biomol. Chem. 2008, 6, 3371-3381. (e) Michrowska, A.; Bujok, R.; Harutyunyan, S.; Sashuk, V.; Dolgonos, G.; Grela, K. NitroSubstituted Hoveyda-Grubbs Ruthenium Carbenes: Enhancement of Catalyst Activity through Electronic Activation. J. Am. Chem. Soc. 2004, 126, 9318-9325. See also ref 12c.

(12) (a) Wang, Z. J.; Jackson, W. R.; Robinson, A. J. An Efficient Protocol for the Cross Metathesis of Sterically Demanding Olefins. Org. Lett. 2013, 15, 3006-3009. For an earlier demonstration of the benefit of using a prenyl vs allyl group in a cross-metathesis with a 1,2disubstituted olefin see: (b) Netscher, T. Preparation of TrialkylSubstituted Olefins by Ruthenium Catalyzed Cross-Metathesis. J. Organomet. Chem. 2006, 691, 5155-5162. (c) Armbrust, K. W.; Beaver, M. G.; Jamison, T. F. Rhodium-Catalyzed Endo-Selective Epoxide-Opening Cascades: Formal Synthesis of (-)-Brevisin. J. Am. Chem. Soc. 2015, 137, 6941-6946.

(13) For the kinetic benchmarking of prenyl (i.e. trisubstituted olefins) in ring-closing metathesis see: Bahou, K. A.; Braddock, D. C.; Meyer, A. G.; Savage, G. P. Kinetic Benchmarking Reveals the Competence of Prenyl Groups in Ring-Closing Metathesis. Org. Lett. 2017, 19, 5332-5335 and references cited therein.

(14) For a recent review on metathesis of terpenes see: Bruneau, C.; Fischmeister, C.; Mandelli, D.; Carvalho, W. A.; dos Santos, E. N.; Dixneuf, P. H.; Sarmento Fernandes, L. Transformations of Terpenes and Terpenoids via Carbon-Carbon Double Bond Metathesis. Catal. Sci. Technol. 2018, 8, 3989-4004.

(15) Diver and co-workers have reported the use of an allyl relay grouping for prenyl alcohol to surmount the unreactive nature of geminal olefins in ene-yne cross metathesis: Clark, J. R.; French, J. M.; Jecs, E.; Diver, S. T. Geminal Alkene-Alkyne Cross Metathesis Using a Relay Strategy. Org. Lett. 2012, 14, 4178-4181.

(16) There is only further limited literature precedent for relay cross metathesis all of which provide conjugated alkene products: (a) Malla, R. K.; Ridenour, J. N.; Spilling, C. D. Relay cross metathesis reactions of vinylphosphonates. Beilstein J. Org. Chem. 2014, 10, 1933-1941. 
For the construction of conjugated Z-enynes (b) Hansen, E. C.; Lee, D. Efficient and Z-Selective Cross metathesis of Conjugated Enynes. Org. Lett. 2004, 6, 2035-2038. (c) Kim, H.; Lee, H.; Lee, D.; Kim, S.; Kim, D. Asymmetric Total Syntheses of (+)-3-(Z)-Laureatin and (+)-3-(Z)-Isolaureatin by "Lone Pair-Lone Pair Interaction-Controlled” Isomerization. J. Am. Chem. Soc. 2007, 129, 2269-2274. (d) Senapati, S.; Das, S.; Ramana, C. V. Total Synthesis of Notoryne. J. Org. Chem. 2018, 83, 12863-12868.

(17) Rao, H. S. P.; Senthilkumar, S. P. A convenient procedure for the synthesis of allyl and benzyl ethers from alcohols and phenols. J. Chem. Sci. 2001, 113, 191-196.

(18) Sharpless, K. B.; Amberg, W.; Bennani, Y. L.; Crispino, G. A.; Hartung, J.; Jeong, K. S.; Kwong, H. L.; Morikawa, K.; Wang, Z. M.; $\mathrm{Xu}, \mathrm{D}$;; Zhang, X.-L. The Osmium-Catalyzed Asymmetric Dihydroxylation: A New Ligand Class and a Process Improvement. J. Org. Chem. 1992, 57, 2768-2771.

(19) Marsico, G.; Pignataro, B. A.; Masi, M.; Evidente, A.; Casella, F.; Zonno, M. C.; Tak, J.-H.; Bloomquist, J. R.; Superchi, S.; Scafato, P. Asymmetric Synthesis and Structure-Activity Studies of the Fungal Metabolites Colletorin A, Colletochlorin A and their Halogenates Analogues. Tetrahedron 2018, 74, 3912-3923.

(20) Ebner, C.; Müller, C. A.; Markert, C.; Pfaltz, A. Determining the Enantioselectivity of Chiral Catalysts by Mass Spectrometric Screening of Their Racemic Forms. J. Am. Chem. Soc. 2011, 133, $4710-4713$.

(21) Chatterjee, A. K.; Choi, T.-L.; Sanders, D. P.; Grubbs, R. H. A General Model for Selectivity in Olefin Cross Metathesis. J. Am. Chem. Soc. 2003, 125, 11360-11370.

(22) Acetonides $\mathbf{5} \mathbf{d}-\mathbf{f}$ were used for these control experiments since truncated epoxide $\mathbf{5 h}$ was found to be too volatile for isolation.

(23) The $E / Z$ ratio of trisubstituted alkenes was determined by ${ }^{1} \mathrm{H}$ NMR integration of selected signals (see Experimental Section). The $E$ isomers were identified as the major isomers on the basis of their characteristically shielded methyl groups of the trisubstituted olefin in the ${ }^{13} \mathrm{C}$ NMR spectrum. See e.g., for $E$ - and Z-8i: Watanabe, Y.; Laschat, S.; Budde, M.; Affolter, O.; Shimada, Y.; Urlacher, V. B. Oxidation of acyclic monoterpenes by P450 BM-3 monooxygenase: influence of the substrate $E / Z$-isomerism on enzyme chemo- and regioselectivity. Tetrahedron 2007, 63, 9413-9422.

(24) All $E / Z$ ReXM products were inseparable by standard silica gel chromatography.

(25) 2,5-Dihydrofuran (bp 66-67 ${ }^{\circ} \mathrm{C}$ ) can be observed by ${ }^{1} \mathrm{H}$ NMR (if the reaction is not purged of volatiles) after direct dissolution of the crude reaction mixtures in $\mathrm{CDCl}_{3}\left(\delta_{\mathrm{H}} 5.87(\mathrm{~s}, 2 \mathrm{H}), 4.62(\mathrm{~s}, 4 \mathrm{H})\right)$. Isobutylene as a more volatile component $\left(\mathrm{bp}-7^{\circ} \mathrm{C}\right)$ is not observed as expected.

(26) For the degradation of precatalyst 1 with primary alcohols see: Dinger, M. B.; Mol, J. C. Degradation of the Second-Generation Grubbs Metathesis Catalyst with Primary Alcohols and Oxygen Isomerization and Hydrogenation Activities of Monocarbonyl Complexes. Eur. J. Inorg. Chem. 2003, 2003, 2827-2833.

(27) Characteristic signals for monocyclized compounds corresponding to cyclic ethers $\left[\mathrm{ca} .10 \%, \delta_{\mathrm{H}} 3.81(\mathrm{~d}, J=5.1 \mathrm{~Hz}, 1 \mathrm{H}), 3.74\right.$ (d, $J=5.4 \mathrm{~Hz}, 1 \mathrm{H}) \mathrm{ppm}, 2$ diastereoisomers] and endocyclic [ca. 8\%, $\delta_{\mathrm{H}} 5.23(\mathrm{br} \mathrm{s}, 1 \mathrm{H})$ and $5.19(\mathrm{br} \mathrm{s}, 1 \mathrm{H}) \mathrm{ppm}, 2$ diastereoisomers $]$ and exocyclic [ca. $10 \%, \delta_{\mathrm{H}} 4.95,4.71,4.87$, and $4.66 \mathrm{ppm}, 2$ diastereoisomers] alkenes were observed in the ${ }^{1} \mathrm{H}$ NMR of the crude reaction mixture, based on previous reports of the corresponding des-methoxy compounds in the literature (see e.g., ref $2 \mathrm{a})$.

(28) Tricycle $\mathbf{1 1}$ has previously only been reported as a racemate (see e.g., ref $2 \mathrm{a}$ ).

(29) (a) ${ }^{1} \mathrm{H}$ NMR, ${ }^{13} \mathrm{C}$ NMR, IR and MS data are available via a data repository as: Bahou, K. A.; Braddock, D. C.; Shi, Z.; He, T. A Relay Strategy Actuates Pre-Existing Trisubstituted Olefins in Monoterpenoids for Cross Metathesis with Trisubstituted Alkenes. Imperial College HPC Data Repository 2019, DOI: 10.14469/hpc/ 5737. (b) An earlier version of this article was deposited to the ChemRxiv preprint server on the 16th December 2019.
(30) Rowley, J. T.; Sharp, I.; Gosney, A. G. Practical Organic Chemistry; Chapman and Hall: New York, 1989.

(31) van Lint, M.; Gümüs, A.; Ruijter, E.; Faber, K.; Orru, R. V. A.; Hall, M. Enantioselective Bio-Hydrolysis of Geranyl-Derived racEpoxides: A Chemoenzymatic Route to trans-Furanoid Linalool Oxide. Adv. Synth. Catal. 2019, 361, 813-825.

(32) Lai, Y.-L.; Huang, J.-M. Palladium-Catalyzed Electrochemical Allylic Alkylation between Alkyl and Allylic Halides in Aqueous Solution. Org. Lett. 2017, 19, 2022-2025.

(33) Konopacki, D. B.; Shortsleeves, K. C.; Turnbull, M. M.; Wikaira, J. L.; Hobson, A. D. Lewis Acid Catalyzed Cyclizations of Epoxidized Baylis-Hillman Products: A Straightforward Synthesis of Octahydrobenzo[e]Azulenes. Eur. J. Org. Chem. 2015, 2015, 54535463.

(34) Shi, X.; Liu, X.; Yang, F.; Wang, Y.; Wang, Z.; Jiao, X.; Xie, P. Asymmetric Total Synthesis of (+)-Isocryptotanshinone and Formal Synthesis of (-)-Cryptotanshinone. Tetrahedron 2019, 75, 39623967.

(35) Mahto, P.; Rana, N. K.; Shukla, K.; Das, B. G.; Joshi, H.; Singh, V. K. Asymmetric Multifunctional Modular Organocatalysis: One-Pot Direct Strategy to Enantiopure $\alpha, \beta$-Disubstituted $\gamma$-Butyrolactones. Org. Lett. 2019, 21, 5962-5966.

(36) Block, E.; Jeon, H. R.; Putman, D.; Zhang, S.-Z. Prepackaged Ramberg-Bäcklund Reagents: Useful Tools for Organic Synthesis. Tetrahedron 2004, 60, 7525-7541.

(37) Takakura, H.; Kojima, R.; Kamiya, M.; Kobayashi, E.; Komatsu, T.; Ueno, T.; Terai, T.; Hanaoka, K.; Nagano, T.; Urano, Y. New Class of Bioluminogenic Probe Based on Bioluminescent EnzymeInduced Electron Transfer: BioLeT. J. Am. Chem. Soc. 2015, 137, 4010-4013.

(38) Amano, T.; Yoshikawa, K.; Ogawa, T.; Sano, T.; Ohuchi, Y.; Tanami, T.; Ota, T.; Hatayama, K.; Higuchi, S.; Amanuma, F.; Sota, K. Synthesis and Antiinflammatory Activity of Alkenylphenylpropionic Acids. Chem. Pharm. Bull. 1986, 34, 4653-4662.

(39) Houjeiry, T. I.; Poe, S. L.; McQuade, D. T. Synthesis of Optically Active 4-Substituted 2-Cyclohexenones. Org. Lett. 2012, 14, 4394-4397.

(40) Vyvyan, J. R.; Brown, R. C.; Woods, B. P. Alkylation of 2Substituted (6-Methyl-2-Pyridyl)Methyllithium Species with Epoxides. J. Org. Chem. 2009, 74, 1374-1376.

(41) Gerbino, D. C.; Mandolesi, S. D.; Schmalz, H.-G.; Podestá, J. C. Introduction of Allyl and Prenyl Side-Chains into Aromatic Systems by Suzuki Cross-Coupling Reactions. Eur. J. Org. Chem. 2009, 2009, 3964-3972.

(42) Tsangarakis, C.; Arkoudis, E.; Raptis, C.; Stratakis, M. Selective Monocyclization of Epoxy Terpenoids Promoted by Zeolite NaY. A Short Biomimetic Synthesis of Elegansidiol and Farnesiferols B-D. Org. Lett. 2007, 9, 583-586.

(43) Xu, D.; Crispino, G. A.; Sharpless, K. B. Selective Asymmetric Dihydroxylation of Dienes. J. Am. Chem. Soc. 1992, 114, 7570-7571. 